\title{
WestVirginiaUniversity
}

THE RESEARCH REPOSITORY @ WVU

Graduate Theses, Dissertations, and Problem Reports

2014

\section{Microfluidic 3D Gradient Generator for Studying Tubulogenesis}

Bhargavi Thotakura

Follow this and additional works at: https://researchrepository.wvu.edu/etd

\section{Recommended Citation}

Thotakura, Bhargavi, "Microfluidic 3D Gradient Generator for Studying Tubulogenesis" (2014). Graduate Theses, Dissertations, and Problem Reports. 6806.

https://researchrepository.wvu.edu/etd/6806

This Thesis is protected by copyright and/or related rights. It has been brought to you by the The Research Repository @ WVU with permission from the rights-holder(s). You are free to use this Thesis in any way that is permitted by the copyright and related rights legislation that applies to your use. For other uses you must obtain permission from the rights-holder(s) directly, unless additional rights are indicated by a Creative Commons license in the record and/ or on the work itself. This Thesis has been accepted for inclusion in WVU Graduate Theses, Dissertations, and Problem Reports collection by an authorized administrator of The Research Repository @ WVU. For more information, please contact researchrepository@mail.wvu.edu. 
Microfluidic 3D Gradient Generator for Studying Tubulogenesis

Bhargavi Thotakura

Thesis submitted to the Benjamin M. Statler College of Engineering and Mineral Resources at West Virginia University in partial fulfillment of the requirements

for the degree of

Master of Science

in

Electrical Engineering

Dr. Yuxin Liu, Committee Chairperson

Dr. Yon Rojanasakul

Dr. Thirimachos Bourlai

Lane Department of Computer Science and Electrical Engineering

Morgantown, West Virginia

2014

Keywords: Microfluidics, Angiogenesis, Tubulogenesis, Extra Cellular Matrix, HUVEC Copyright 2014 Bhargavi Thotakura 


\title{
ABSTRACT \\ Microfluidic 3D Gradient Generator for Studying Tubulogenesis
}

\author{
Bhargavi Thotakura
}

Tubulogenesis and Angiogenesis is an important process in the formation of new blood vessels, and play a vital role in many physiological and pathological processes, such as cancer metastasis. To study this process in the laboratory, versatile, reliable and affordable assays are essential. The objective of this thesis work was to develop a microfluidic three-dimensional (3D) gradient device, which embedded collagen within the device for generating chemical gradient through it, and investigated it as a useful research tool for angiogenesis study with endothelial cells forming branching tubes inside the collagen.

With the enormous growth of microfluidic technologies, the scope for creating more realistic in vitro cell angiogenesis assays that replicate many aspects of the true in vivo microenvironment has increased. Although few conventional assays like rabbit ear chamber assay and chick chorio allantoic membrane assay are available in market, they set their own limitations for further studies. For instance, high in cost, lack of precise gradient control and characterization, limitation in mimicking the micro environment etc. Here, in this thesis work we introduced a microfluidic 3D gradient device and angiogenesis study assay that serves as a versatile single platform for the study of angiogenesis which is crucial step in the study of vascular biology and it related diseases. Briefly, a uniform collagen layer, which served as an extra cellular matrix (ECM), gave a precise control of generating a chemical gradient over a long period of time and offered an excellent monitoring capability with response to observing endothelial cells' formation of tube structures. Human umbilical vein endothelial cells (HUVEC) were cultured inside the corresponding location of a micro channel for 4-5 days, and their responses to the quantified gradient of vascular endothelial growth factor (VEGF) were examined. Immunofluorescence staining and confocal imaging were made for detailed study on formed tubing. These results suggested that the microfluidic 3D gradient device can conveniently generate a stable chemical gradient and provide an easy way for the study of angiogenesis. 
Dedication

\section{To My Beloved Family}




\section{ACKNOWLEDGMENT}

I would like to take an opportunity to thank all the great individuals for their utmost support in fulfilling my journey as a Graduate Student with much more worthwhile memories.

I express my heartfelt reverence to my advisor Dr.Yuxin Liu for her constant Guidance, Support, Motivation and Freedom which indeed is, extremely beneficial to me in all ways. I will always remember her soothing words, "Be Patient You Will Get It" during my times of depression, which really makes me to regain all my positive strength to go on! Her in-depth knowledge in the field of microfluidics has been highly impressive and makes my work really productive.

I would like to express my deepest gratitude to Dr.Yon Rajanasakul for helping me using his lab forx cell culturing which plays a key role for my experiments. I also like to thank Dr.Warangkana Lohcharoenkal for teaching me all cells related stuff, I really appreciate her patience as sometimes I completely messed up everything while working with her. Her comments and questions about the process are quite challenging and it was from her I learned to answer and question myself $W H Y$ ? Before proceeding further.

I would like to thank Dr.Thirimachos Bourlai for his valuable suggestions and thanks for allocating time for me even in your busy hours.

My sincere thanks to Dr.Kolin Brown and all clean room facilities crew. It was from clean room my initial step of research begins and thanks a lot for making that step as strong and as deep to move forward. 
As a research student my clock in lab hides more hours than at home. So it is very essential to have a healthy and friendly environment around. I am glad to have been surrounded with many lovely hearts and brains. Thanks to Xiang Li, Hanyuan Zhang, Zhouchun Huang and Loukhya Kakumanu for your continuous support, interesting comments and ideas during my stay. Many steps in my research are filled with your invaluable ideas and suggestions. During my stay in Morgantown, life may not be such lively and colorful without one word 'FRIENDS'. They are my Energy boosters, Supporters, Entertainers and Therapists. Actually it was because of them I rarely feel like missing home. At this context I am extending my hands to thank Lekha, Kavitha ,Neeharika, Harika, Sravanthi, Pallavi, Spandana, Meghana and all wonderful juniors around. This stay may not be adventurous and fun without Crazy and awesome People. Thanks to Hari, Vamsi, Saurabh, Satish, Pradeep, Sujan, Murthy, Yaswanth, Raj, Krishna, Harish and all for making me feel rejoicing during my dull phase. (Sorry if I am missing any)

I can't ever imagine my current position without the love, support and selfless sacrifices of my family. Being hailed from a very conservative and rural background they strived every second to educate me and my brother. Words seem to be inadequate to express my love towards them. Mom and Dad thanks a lot for everything and I promise to keep generous meaning to this life given by you. Janak Varma (my brother) thanks for being my blood best friend, teaser, and appreciator. I also like to thank all my Aunts, Uncles and cousins for providing me immense moral support during my stay away from home. 


\section{TABLE OF CONTENTS}

MICROFLUIDIC 3D GRADIENT GENERATOR FOR STUDYING TUBULOGENESIS I

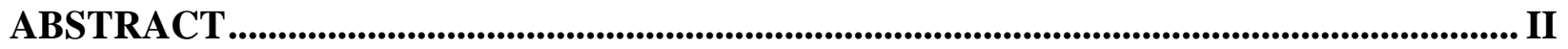

DEDICATION...................................................................................................................................II

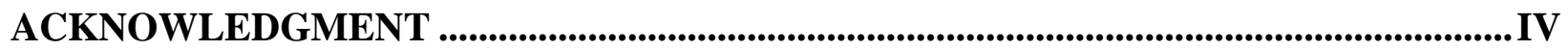

TABLE OF CONTENTS _..........................................................................................

LIST OF FIGURES ..........................................................................................................VIII

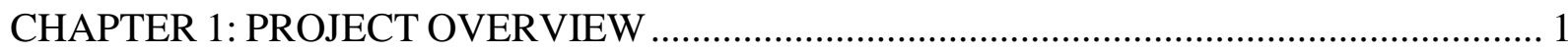

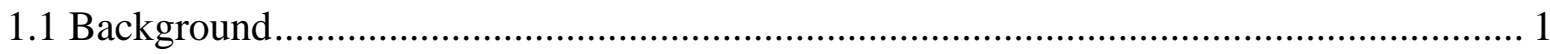

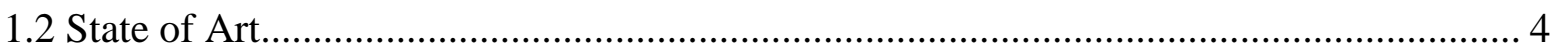

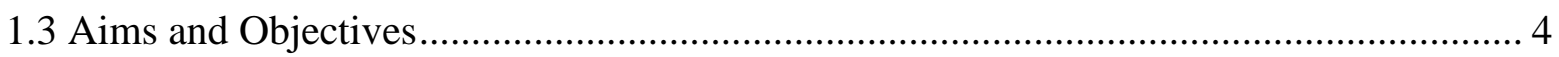

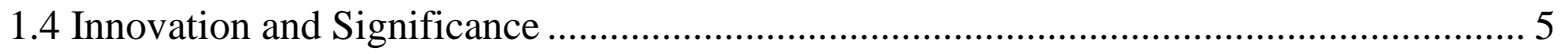

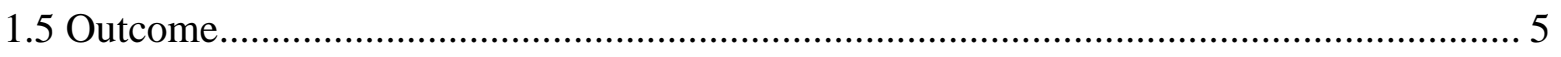

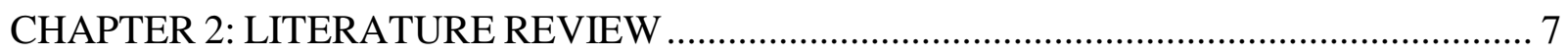

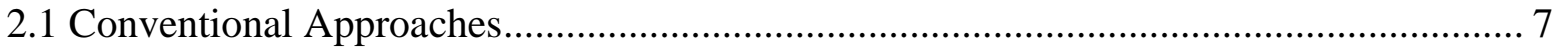

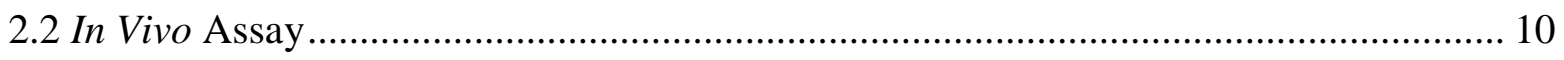

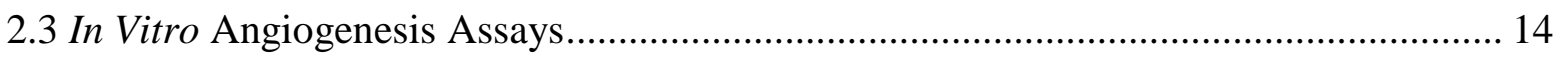

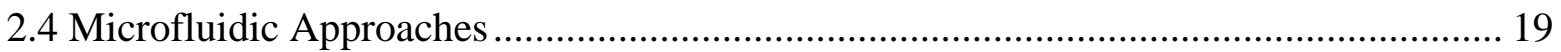

CHAPTER 3: MICROFLUIDIC 3D GRADIENT ASSAY DESIGN ...................................... 23

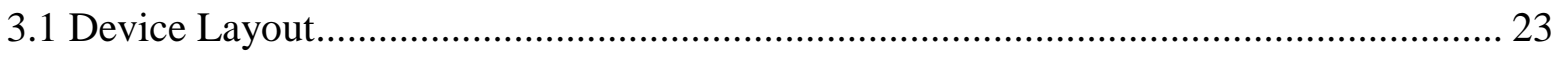

3.2 Microfluidic Gradient Device Design and Specifications ............................................... 24

3.3 Current Model for the Study of Tubulogenesis ............................................................. 26

CHAPTER 4: MICROFLUIDIC CHIP FABRICATION ……………................................ 28

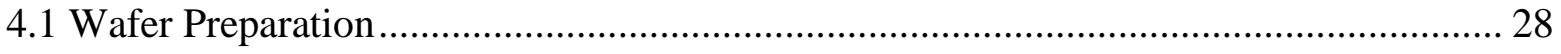

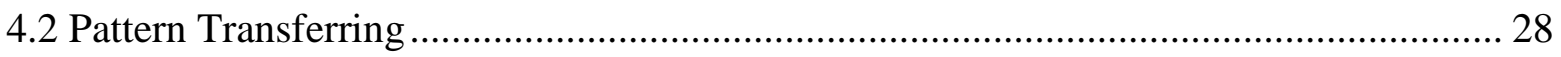

4.3 Single Layer SU-8 Fabrication using UV Flood Exposure ……………………............ 31

4.4 Double Layer SU8 Fabrication using Mask Aligner ........................................................ 33

4.5 Potential Problems and Solutions ........................................................................... 37

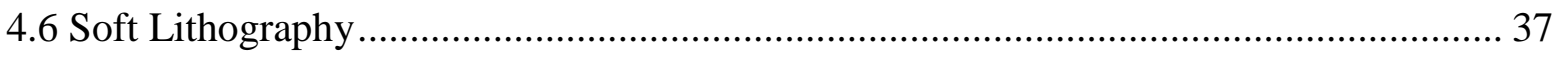


4.7 Potential Problems and Solutions ..................................................................... 40

CHAPTER 5: CELL CULTURE IN MICROFLUIDIC DEVICES …................................ 41

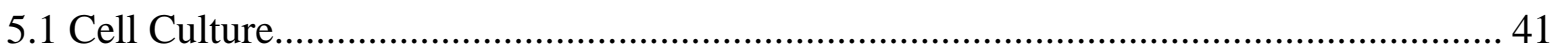

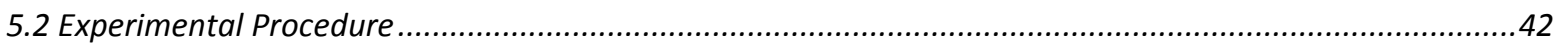

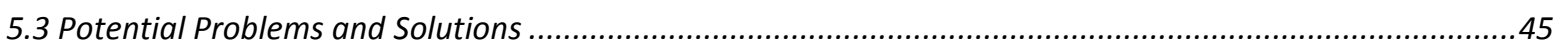

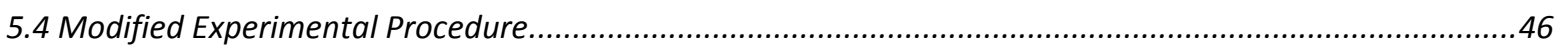

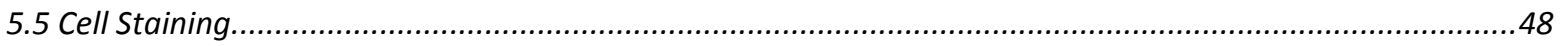

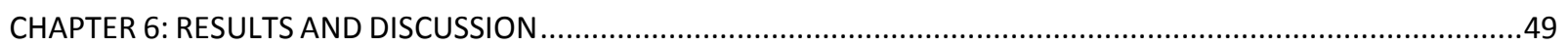

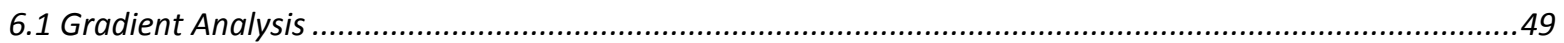

6.2 Formation of Tubulogenesis inside Microfluidic Device ....................................................................51

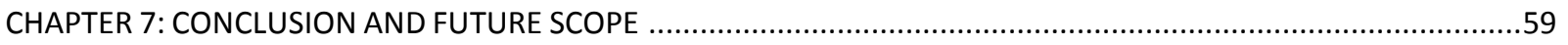

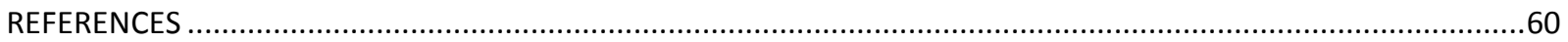




\section{LIST OF FIGURES}

Figure 1 Cancer cell division and formation of tumor (Ref from cancer help UK). .................. 1

Figure 2 Cytoskeletal model of cell migration................................................................. 2

Figure 3 (A) angiogenesis: it occurs initially with the formation of the budding (1) or endothelial cell outgrowth followed by stretching (2) of small vessels and branching by proliferation (3) of

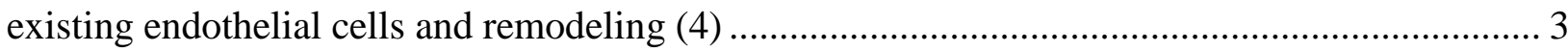

Figure 4 Sequence of steps in angiogenesis by sprout formation (Adapted from refs )............. 8

Figure 5 The appearance of the CAM in the absence (A) or presence (b) of thymidine phosphorylase. Photographs courtesy of Dr Roy Bicknell, CRUK, Oxford. ........................... 10

Figure 6 The dorsal skinfold window chamber model using in vivo microscopy ${ }^{12}$................. 11

Figure 7 Tube-formation assay, HUVECs $(2 \times 104$ cells/well $)$ were plated on Matrigel-precoated 96-well plates and treated with different concentrations of EPMC for 24 hours. ${ }^{14}$................... 15

Figure 8 Sequences of steps followed by Aortic assay to study angiogenesis ........................ 15

Figure 9 Effect of U94/REP on microvessel outgrowth in vitro. Chick aortic rings were

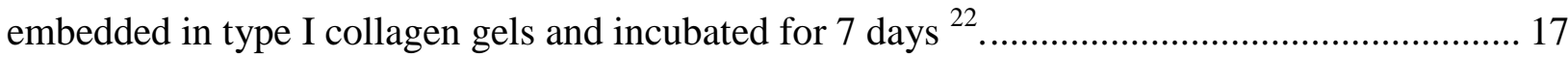

Figure 10 3D in vitro model for HUVEC cell angiogenesis ............................................... 20

Figure 11 A. The microfluidic-based cell culture platform directs vascular growth along an alginate bead scaffold to form a 3D capillary bed, B,C. Microfluidic devices cultured under static and flow conditions D, Growth of HUVEC cells sprouting after 3 days growth ..................... 21

Figure 12 Microfluidic photomask layout using AutoCAD and Photomask........................... 23

Figure 13 AutoCAD layout and specifications of a 3D gradient device. ............................... 24

Figure 14 AUTOCAD model with curvy surfaces and pillars......................................... 25

Figure 15 Modified AutoCAD design and Maximized image of the middle channel with pillars

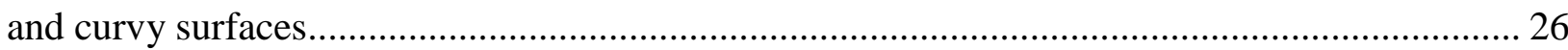

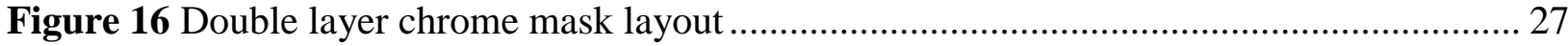

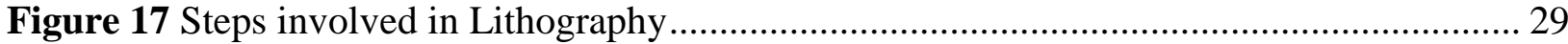

Figure 18 Karl Suss 1000 UV intensity meter and probe and Front panel of exposure timer..... 32

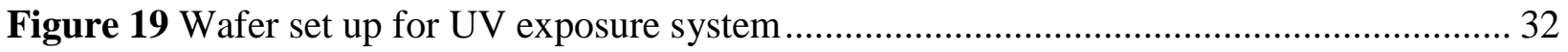

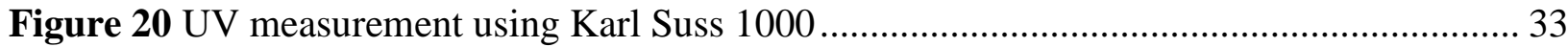

Figure 21 Mask Aligner (White hall clean room , WVU) ................................................ 34 
Figure 22 Pattern washed away due to over development

Figure 23 Schematic illustration of the procedure for casting PDMS replicas from a master

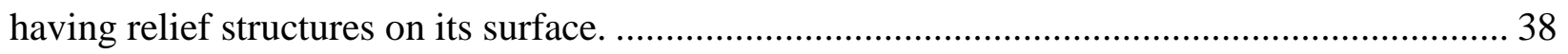

Figure 24 Demolding PDMS wafer from Master Mold........................................................ 39

Figure 25 Experimental set up for collagen loading using syringe pumps ............................. 44

Figure 26 Fluorescence image of generated gradient across the collagen channel. .................. 50

Figure 27 FITC-albumin gradient across the width of the channel, a stable linear gradient

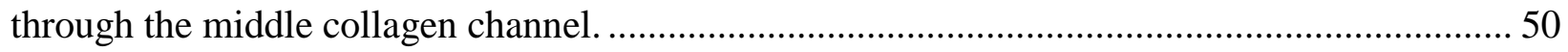

Figure 28 HUVEC cells loaded in the top channel and migration of cells in correspondence to

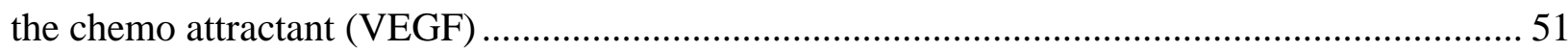

Figure 29 Analysis of control group cells, inside the microfluidc device where there are no traces of tubing formation. 52

Figure 30 Formation of tubogenesis of HUVEC cells in the experimental group( with VEGF)

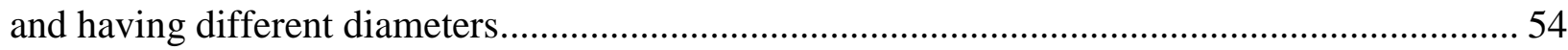

Figure 31 Longitudinal tube formation inside the micro channel, around a micro pillar........... 55

Figure 32 Bifurcation of cell sprouting and formation of tube, around a micro pillar ............... 56

Figure 33 Formation of Lumen, around a micro pillar. ...................................................... 57

Figure 34 Formation of multiple tubes into the collagen scaffold from the HUVEC monolayer. 


\section{CHAPTER 1: PROJECT OVERVIEW}

\subsection{Background}

There is a remarkable increase in cancer deaths over past few decades. According to the report provided by World Cancer Research and Fund International (WCRF) there are almost 14 million cancer cases around the world in the year 2012.The number is expected to increase to 24 million by 2035 . So there is an urgent need to focus more and more on cancer related research. So, the best way to handle this problem is to begin and understand from cancer biology. For instance, by having a clear idea on how cancer cell survival and growth are controlled, there might be scope to predict how tumor cells undergo natural selection to avoid drug's effect and stop reacting to the treatment.

'Cancer' is a disease characterized by unregulated growth of cells. When these cells make billions or trillions of copies of the original cancerous cells and forms 'Tumor'. Scientists describe cancer cells as 'immortal'.

Cancer Cells and formation

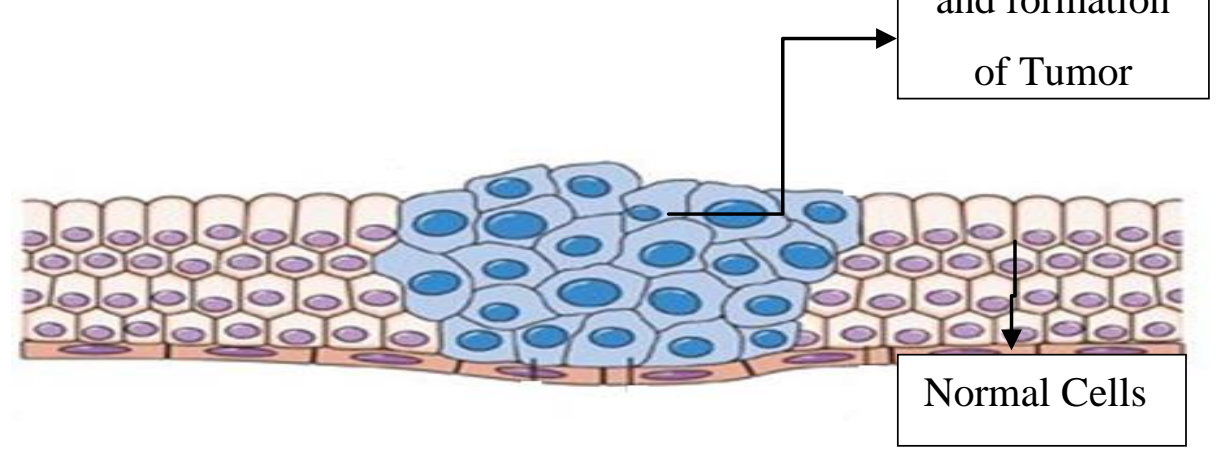

Figure 1 Cancer cell division and formation of tumor ${ }^{1}$. 
There is no one single cause for cancer. Scientists believe that it is the interaction of many factors together that produce cancer. The factors involve may be genetic, environmental, or constitutional characteristics of the individual.

When a tumor successfully spreads to other parts of the body and grows, invading and destroying other healthy tissues, it is said to have metastasized. This process itself is called metastasis, and the result is a serious condition that is very difficult to treat. In the process of metastatics, cell migration and angiogenesis plays a vital role.

Cell Migration is defined as the translation of cells from one location to another. It is essential for many physiological and pathological processes, including new blood vessel formation, wound healing and inflammation. Cell migration is traditionally viewed as a mechanical process where the primary driving force of migration is remodeling of the actin cytoskeleton (Wang, 1985). Here the action rich protrusions at the font push the cell forward which is shown in figure(2).

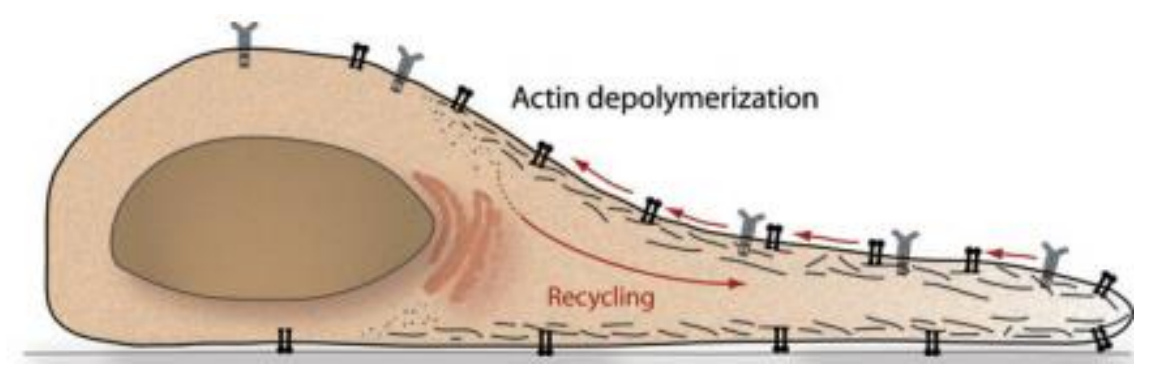

Figure 2 Cytoskeletal model of cell migration.

In the cytoskeletal model polymerization of actin filaments at the leading edge pushes the cell forward while the rear is contracted through forces generated by actomyosin. This movement is not random, but directed by extracellular guidance cues which can be presented as gradients either bound to the extracellular matrix (haptotaxis) or as soluble growth factors (chemotaxis). 
Cell angiogenesis - the formation of microvessels from the existing vessels- is an important factor in cell biology (Figure 3). ${ }^{1}$ Angiogenesis occurs through looping or sprouting. It is the most common method to create new vessels. In addition on being normal it sometimes helps for the abnormal growth results in tumor.

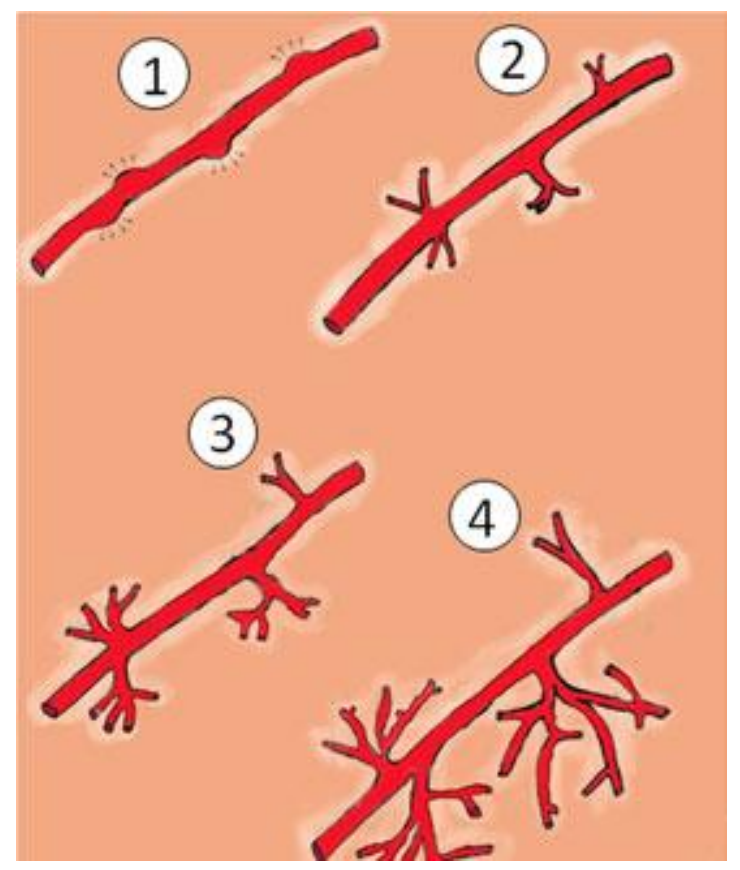

Figure 3 Angiogenesis ${ }^{5}$ : it occurs initially with the formation of the budding (1) or endothelial cell outgrowth followed by stretching (2) of small vessels and branching by proliferation (3) of existing endothelial cells and remodeling (4)

Tumors have high metabolic needs which are greater than the preexisting vascular network can provide, for tumors to develop in size and metastatic potential they must induce angiogenesis within them. They achieve this by secreting growth factors such as Vascular Endothelial Growth Factor (VEGF) and Fibroblast Growth Factor (FGF). Apart from this shear stress ${ }^{2,3}$, intestinal flow $^{4,5}$ and matrix stiffness ${ }^{6}$ also play key role in sprouting angiogenesis. Clear discussion of these concepts is practically analyzed in the later chapters. 


\subsection{State of Art}

Before the development of the first in vitro assay, several attempts had been made to mimic the in vivo environment. Later with excessive research few assays (Conventional) were developed and proven their efficient functionality. However, these conventional assays cannot bury the gap i.e. the need for quantitative assays, 3D analysis and real time continuous flow control and image capturing ${ }^{2,7,8}$ still remains as a problem to concentrate. In vitro culture platforms designed to model and measure these tubing and vessel formations are more time consuming, high in cost and its precise control and characterization possesses several challenges like poor control of cell biology, high in cost and harder analysis etc.

Recent advancements in micro fabrication technologies have provided a new ray of scope with new quantitative methods ${ }^{9}$, that are versatile, cost effective and better control for studying endothelial cell biology, including angiogenesis ${ }^{10,11}$. Microfluidic cell migration devices provides a precise control of gradient and also imposes a stable linear gradient. Apart from this, it also provides excellent real time imaging, which helps to regain better quantification ${ }^{12}$.

\subsection{Aims and Objectives}

The main objective is to design microfluidic device using Photolithography and soft lithography techniques which is aimed to work on 3D analysis with collagen-I scaffold to mimic the Extra Cellular Matrix (ECM) through which the cells proliferate and migrate ${ }^{6,9}$. collagen and hydrogel scaffolds are extensively used in the studies of 3D cellular morphogenesis, angiogenesis, and cancer metastasis. This microfluidic device is used in the study of cell angiogenesis (formation of micro tubes followed by migration), Human umbilical vein endothelial cells (HUVEC) are used to test this approach. Major agenda is to culture HUVEC 
monolayer and by manually loading chemo attractant (VEGF), analyze cell new micro vessels formation up to desired amount of time period (3-4 days).

\subsection{Innovation and Significance}

Study of cell migration and angiogenesis is significant as it leads to cancer metastatics. Among many in vitro approaches microfluidic technology proves as a powerful tool to use because of its higher experimental throughput, easy controlled environment followed by reliable and inexpensive approach.

The microfluidic device fabricated in this experiment, generates a linear gradient - an indication of uniform flow of gradient: which can be easily visualized with the usage of fluorescence particles as stand-ins for bioactive molecules without modelling the spatial interruptions. In order to have clear replica of the real environment a three channel concept is used in the design of this device.

Various design considerations are tested during designing this model. For instance, in order to hold the loaded collagen, small and consistently spaced pillars are incorporated throughout the middle of the device. Several possible issues like easy filling, no breakage of collagen etc. were taken into account and correspondingly the modifications were made in the device.

\subsection{Outcome}

The desired response from this microfluidic device is to generate a linear gradient with the presence of collagen I layer and to analyze the formation of micro tubing into the collagen from the HUVEC cells, which will be loaded on one of the chambers. These cells are expected to sense the gradient generated by the chemo attractant (VEGF 50ng/ml) and should spread out to form tube like structures,(angiogenesis) after 3-4 days culture. Later cell staining and confocal 
imaging will be followed in order to confirm the micro tubing formation and to study about the morphology, specifications and number of cells involved in the tubing.

In the later part of the document:

Chapter 2 provides a brief idea on the literature which includes the discussion of conventional and other microfluidic approaches.

Chapter 3 deals with the design specifications of the microfluidic device used in the AutoCAD.

Chapter 4 includes the single layer and double layer fabrication process of photolithography and then followed by the soft lithography process.

Chapter 5 deals with the type of cells used in the experiment, cell culture and the procedure of loading cells to the device followed by cell staining.

Chapter 6 shows the gradient profile and obtained tubulogenesis formation using the microfluidic devices along with discussion.

Chapter 7 shows the highlights in the form of conclusion and future scope. 


\section{CHAPTER 2: LITERATURE REVIEW}

\subsection{Conventional Approaches}

As growing endothelial cells has been a long term challenging problem, because being primary cell line which is indeed hard to culture in flasks, there is a need for the development of agents in order to combat angiogenic diseases like cancer etc. that quantify the neovasculature. Folkamn's group in 1980, after extensive research designed an appropriate protocol, which elucidates the functional/sequential steps in angiogenesis ${ }^{13}$. Despite rapid progress after this protocol, several assays came to limelight, however many unanswerable questions like the molecular mechanism required to switch a cancer cell into angiogenic phenotype? the optimal dosage of various agents etc. are yet to answer. Although there are various comprehensive reviews need to be considered while developing an assay, there is a need to outline the steps in new vessel formation as well as to characterize angiogenetic response.

Before analyzing the in vitro and in vivo assays the understanding of angiogenesis mechanism is prominent.

\subsubsection{Steps involved in Angiogenesis Mechanism}

Even though the cell molecular mechanisms responsible for the angiogenic phenotype are not well known, the sequence of events is well articulated ${ }^{14,15}$. The vascular growth entails endothelial sprouting ${ }^{15}$ or intussusception ${ }^{16}$.The sequence of steps are as illustrated below followed by the corresponding figures ${ }^{17}$. 

a) Dissolution of the basement membrane of the vessel.
b) Migration of the endothelial cells towards the stimulus.
c) Proliferation of the endothelial cells.
d) Formation of lumen (canalization) in the endothelial sprout.
e) Formation of branches and loops by confluence of sprouts to permit blood flow.
f) Investment of vessel with pericytes
g) Formation of basement membrane around the immature vessel.

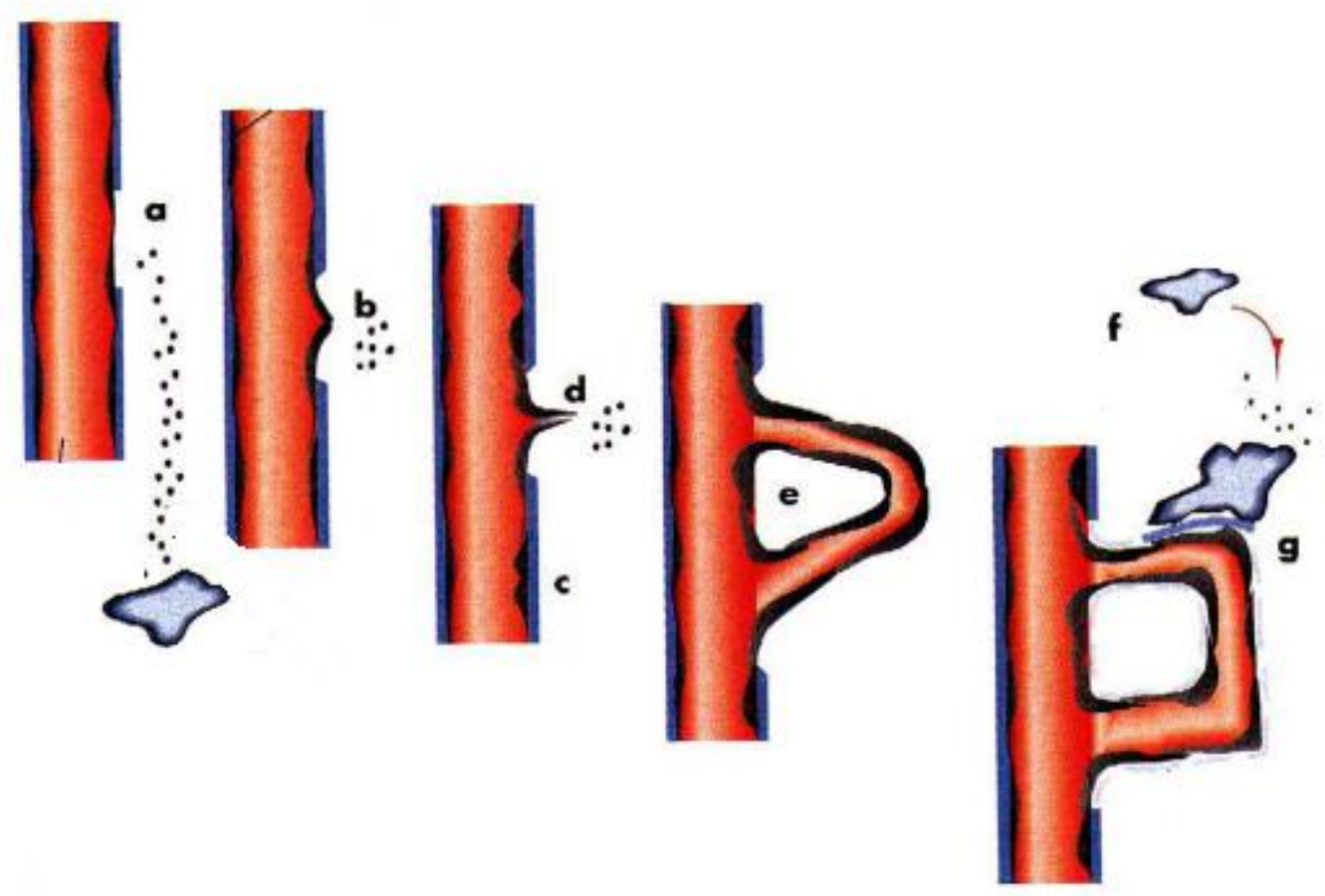

Figure 4 Sequence of steps in angiogenesis by sprout formation ${ }^{6}$

The in vivo angiogenesis assays can be categorized into three main groups:

1. Microcirculatory preparations like rabbit-ear chamber and CAM assay.

2. Vascularization into biocompatible polymer matrix implants like the sponge models.

3. Excised vascularized animal tissue like - corneal micro pocket assay. 
To analyze the mechanisms underlying normal and pathological angiogenesis, many in vivo angiogenic assays have been established to employ different species of laboratory animals, including mammals (mouse, rat, hamster, and rabbit), birds (chicken and quail), and fish (mainly zebra fish).Micro circular preparations allow in vivo observations using light microscopy. Depending on the particular thickness different illuminations are used.

Angiogenic activities can be assessed using both in vitro and in vivo assays. In vivo assays may better mimic angiogenesis as it occurs in normal and pathologic states, but in vitro assays offer several critical advantages than in vivo counterparts. In vitro assays allow identification of direct effects on endothelial cell function, whereas in vivo assays involve multiple cell types and potential metabolic processing of the agent, complicating analysis of the agent's mechanism of action $^{41}$. In vitro assays allow analysis of isolated processes that contribute to angiogenesis whereas in vivo assays model angiogenesis as a whole. In vitro assays allow analysis of variables such as matrix components in isolation; tissue effects and other complexities of environment contribute to angiogenesis in vivo. In vitro assays do not require the technical expertise in animal handling required for in vivo assays. In vitro assays are typically less expensive than in vivo assays, and may be adapted for large-scale screening ${ }^{22}$. Many in vitro angiogenesis assays can be more easily quantified than vessel outgrowth in vivo, and for some assays this quantification can be automated. Finally, in vitro assays allow genetic manipulation of the endothelial cells by transfection or adenoviral infection, as well as utilization of cells and tissue from transgenic mice for stem cell and organ culture assays. Various in vitro and in vivo techniques used to assess various aspects of angiogenesis, are described below. 


\subsection{In Vivo Assay}

\subsubsection{The chick chorio allantoic membrane (CAM) assay:}

The chick chorioallantoic membrane (CAM) assay is probably the most widely used in vivo assay for studying angiogenesis ${ }^{12}$. The test substance is prepared either in slow-release polymer pellets, absorbed by gelatin sponges, or air-dried onto plastic discs; these are then implanted onto the CAM through a window cut carefully in the eggshell. The lack of a mature, immune system in 7-8-day old chick embryos allows for the study of tumor-induced angiogenesis ${ }^{18}$ The angiogenic effects can be measured by counting the number of blood vessels in a given area using a stereomicroscope. In a variation of the CAM assay, shell-less embryos are cultured in Petri dishes prior to applying the test this allows for the quantification of blood vessels over a wider area of the CAM.

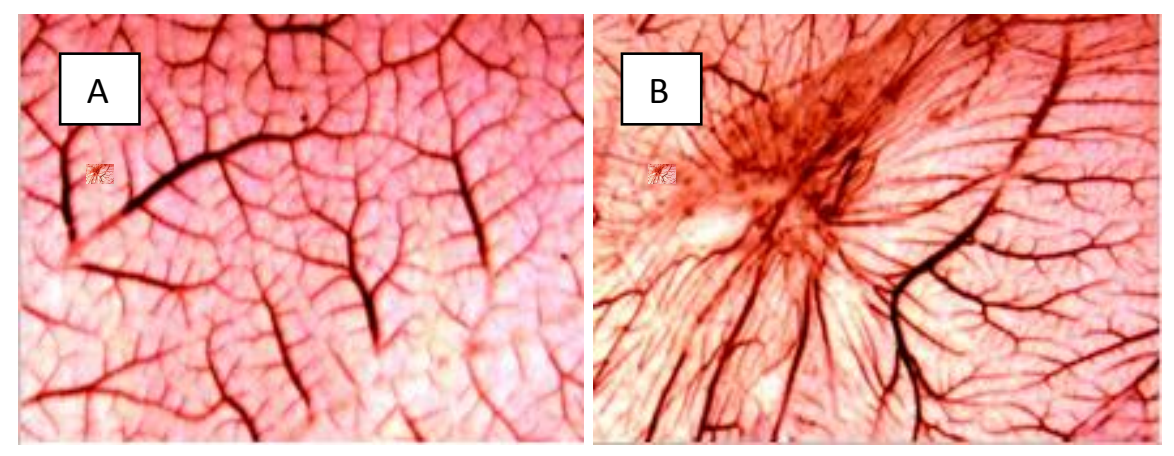

Figure 5 The appearance of the CAM in the absence (A) or presence (B) of thymidine phosphorylase ${ }^{19}$

The CAM assay is a relatively simple and inexpensive in vivo assay suitable for large-scale screening. However, the CAM itself has a well-developed vascular network, thus making it difficult to distinguish new capillaries from existing ones. Furthermore, the 7-8-day old CAM often develops an inflammatory response to a variety of irritants, including shell dust generated when cutting the window in the shell, which can also hamper the identification of new 
vasculature ${ }^{19}$ It is usually necessary to wait for 3 days after making the window before adding the test substance, to check for any immune response. Finally, the membrane is very sensitive to changes in oxygen tension, making the sealing of the window a vital part of the procedure. ${ }^{20}$

\subsubsection{Dorsal air sac model}

The dorsal air sac model is used to examine the in vivo effect(s) of substances against the angiogenic response triggered by cancer cells ${ }^{21}$ Briefly, both sides of a Millipore ring are covered with filters $(0.45 \mu \mathrm{m}$ pore size $)$ and the resultant chamber carefully filled with a tumor cell suspension; this is then implanted into the preformed dorsal air sac of an anaesthetized mouse. Following treatment with the compound of interest, the chamber is carefully removed and rings of the same diameter placed directly upon the sites that were exposed to a direct contact with the chamber. The angiogenic response can then be assessed by counting the number of newly formed blood vessels that lie within the area marked by the ring, using a dissecting microscope.

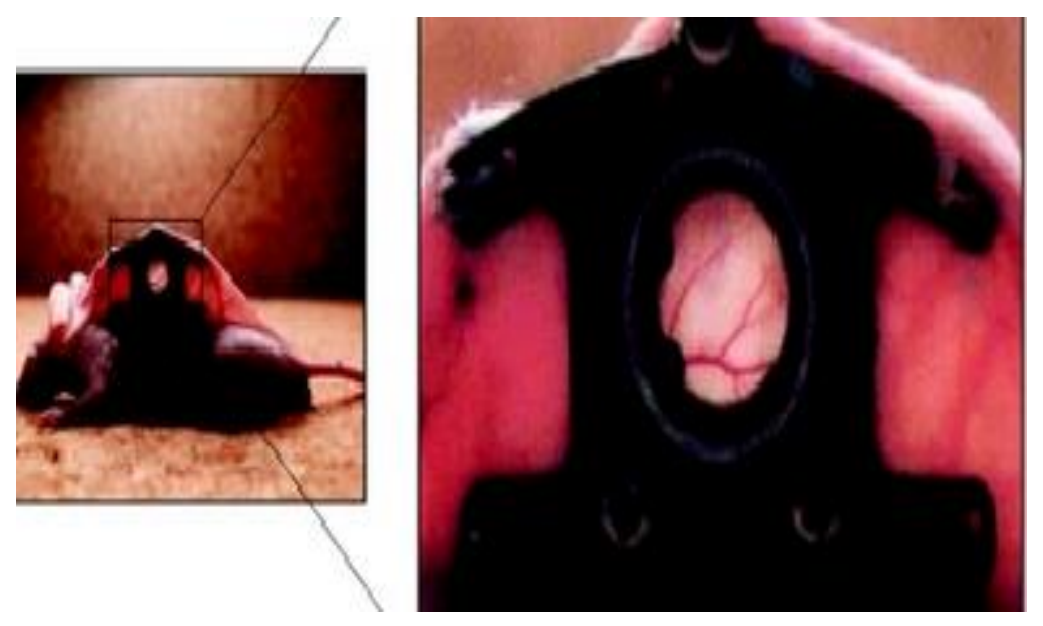

Figure 6 The dorsal skinfold window chamber model using in vivo microscopy ${ }^{12}$ 
This assay is relatively simple to perform, although care must be taken not to irritate the surface upon which the chamber is placed, as this may itself induce angiogenesis and hence mask those blood vessels formed due to the presence of the tumor cells.

\subsubsection{Chamber assays}

The in vivo study of chronic angiogenesis has been greatly advanced by the development of several types of transparent chamber, such as the rabbit ear chamber, dorsal skinfold chamber and cranial window chamber. In these systems, a piece of skin (ear and skinfold chambers) or part of the skull (cranial window chamber) is removed from an anaesthetized animal. Tumor cells, or a gel containing angiogenic factors, is then placed on the exposed surface and covered by glass, which is then secured in place; once the animals have recovered, these models allow for the continuous measurement of various parameters in living animals, including gene expression, angiogenesis, $\mathrm{pH}$ and blood flow ${ }^{22}$ and hence aid the study of the effect of tissue microenvironment on angiogenesis.

Chamber assays allow for the determination of 3D vessel growth in one animal, typically over a period of 1-3 weeks. As a result, separate groups of mice are not required at each measurement point, and hence, the number of animals used is minimized. The cranial window chamber assay has several other advantages including better transplantability and induction of a rapid angiogenic response ${ }^{23}$ However, all chamber assays are invasive and technically demanding. The rabbit ear chamber is expensive for routine use, and a period of 4-6 weeks must pass after surgery before the test substance can be placed into the chamber. The skinfold chamber can have 
poor optical properties due to skin thickness; and the cranial window requires the injection of a fluorescent marker in order to image new vessels.

\subsubsection{The corneal angiogenesis assay}

This assay is considered one of the best in vivo assays, inasmuch as the cornea itself is avascular. Thus, any vessels seen in the cornea after stimulation by angiogenesis-inducing tissues

or factors are new vessels. Even though the original method was developed for rats ${ }^{24}$, later it was adapted to mice ${ }^{25}$ and other frequency used test animals.

The procedure is a pocket is made in the cornea, and test tumors or tissues, when introduced into this pocket, elicit the ingrowth of new vessels from the peripheral limbal vasculature. For test slow release materials such as ELVAX (ethylene vinyl copolymer) or Hydron have been used. To test inhibitors of angiogenesis, one can monitor the effect of such inhibitors on the locally induced angiogenic reaction in the cornea (e.g., by FGF, VEGF, or tumor cells).Later the vascular responses are monitored using a simple stereomicroscope. Methods for quantification also include measuring the area of vessel penetration, the progress of vessels toward the angiogenic stimulus over time, or in the case of fluorescence, histogram analysis or pixel counts above a specific (background) threshold.

The major advantages to the corneal angiogenesis assay: the ability to monitor progress of angiogenesis, the absence of an existing background vasculature in the cornea, and the ability to use mice as experimental animals. On the other hand, the surgical procedure is demanding, so that relatively few animals ( $\sim 20$ mice $)$ can be grafted at a single setting. In addition, the space 
available for introducing test material is limiting, inflammatory reactions are difficult to avoid, and the site, although ideal for visualization, is atypical precisely because the cornea is avascular.

\subsubsection{The Matrigel plug assay}

In contrast to the mouse corneal angiogenesis assay, which requires considerable technical skill, the Matrigel plug assay is pretty easy to handle. Here the procedure includes injection of test cells subcutaneously, to form a plug. This plug can be recovered after 7-21 days in the animal and examined histologically to determine the extent to which blood vessels have entered it. Quantification can also be achieved by measuring the amount of hemoglobin contained in the plug ${ }^{26}$.

Later new modifications were made and the sponge/Matrigel assay came into life. Here Matrigel alone is first introduced into the mouse. A sponge or tissue fragment is then inserted into the plug. New vessels can then be measured by injection of FITC-dextran as described for the corneal assay. The greatest disadvantage of the sponge/Matrigel assay is that it is more timeconsuming than the standard Matrigel plug assay.

\subsection{In Vitro Angiogenesis Assays}

One of the most specific tests for angiogenesis is the measurement of the ability of endothelial cells to form three-dimensional structures (tube formation). This formation can be enhanced by collagen or fibrin coating to the dishes. Tube formation on these coatings is reasonably faithful to the in vivo situation, and the formation of tight junctions can be confirmed by electron microscope. As matrigel and collagen can evoke endothelial cell tube formation ${ }^{18}$ within $24 \mathrm{~h}$, tube formation assays have achieved a prominent place in the array of angiogenesis measures. 

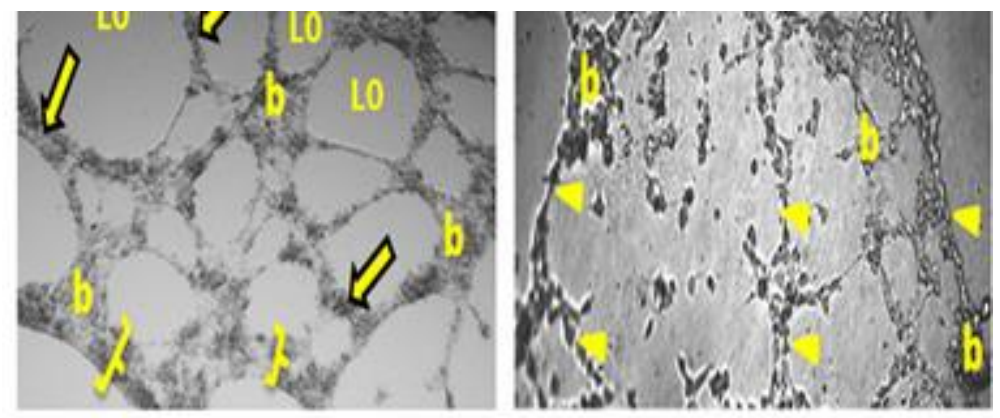

Figure 7 Tube-formation assay, HUVECs $(2 \times 104$ cells/well $)$ were plated on Matrigel-precoated 96-well plates and treated with different concentrations of EPMC for 24 hours. ${ }^{14}$

\subsubsection{The Aortic ring assay}

Nicosia and Ottinetti were the first to devise the rat aortic ring assay in 1990 to assess angiogenesis $^{14 .}$ In vivo angiogenesis involve not only endothelial cells but also their surrounding cells has more recently led to a move to assess angiogenesis by organ culture methods. Of these, the rat aortic ring assay has become the most widely used. In it, the isolated rat aorta is cut into segments that are placed in culture, generally in a matrix-containing environment such as Matrigel. Over the next 7-14 days, the explants are monitored for the outgrowth of endothelial (and other) cells as this is affected by the addition of test substances. Quantification is achieved by measurement of the length and abundance of vessel-like extensions from the explant. The overall study may require 6-9 days and the sequence of steps are as indicated in the figure(8).

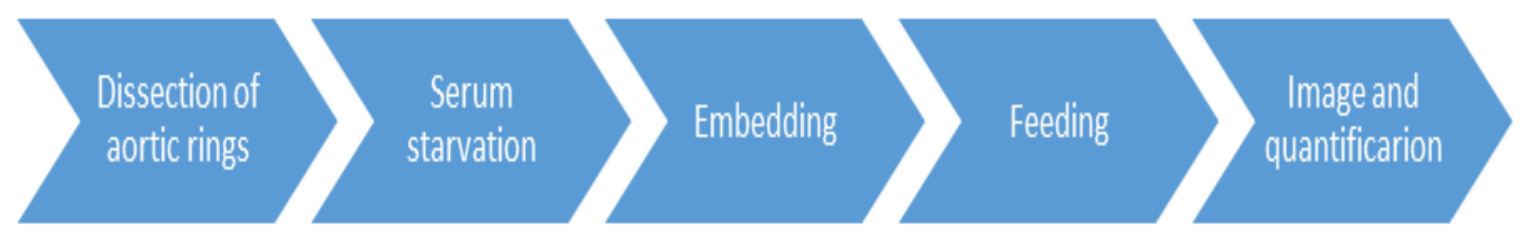

Figure 8 Sequences of steps followed by Aortic assay to study angiogenesis 
This in vitro assay system is considered by many to come closest to simulating the in vivo situation, not only because it includes the surrounding non endothelial cells but also because the endothelial cells have not been preselected by passaging and thus are not in a proliferative state at the time of explantation ${ }^{16}$ and thus more representative of the real-life situation. On the other hand, angiogenesis is primarily a microvascular event, making the aorta a less than an ideal choice.

\subsubsection{The chick aortic arch assay}

This assay represents as a major modification for the rat aortic ring assay. Originally developed for the specific purpose of testing thalidomide (which had previously been shown to have limited effects in rodents but strong effects in chick embryos), the assay avoids the use of laboratory animals, is rapid with an assay time of 1-3 days, and can be carried out in serum-free) medium $^{27}$.

Aortic arches are dissected from day 12-14 chick embryos and cut into rings similar to those of the rat aorta. When the rings are placed on Matrigel, substantial outgrowth of cells occurs within $48 \mathrm{~h}$, with the formation of vessel-like structures readily apparent.

If the aortic arch is averted before explanting, the time can be reduced to $24 \mathrm{~h}$. Both growthstimulating factors, such as FGF-2, or inhibitors, such as endostatin, can be added to the medium, where their effect becomes easily measured. Figure (90) represents the total explant area detailed outgrowth area, in which endothelial cells are seen to form tube like structures. 
Quantification of endothelial cell outgrowth in both the aortic ring and the aortic arch cultures can be achieved by the use of fluorescein-labeled lectins such as BSL-I and BSL-B4 or by staining of the cultures with labeled antibodies to CD31.
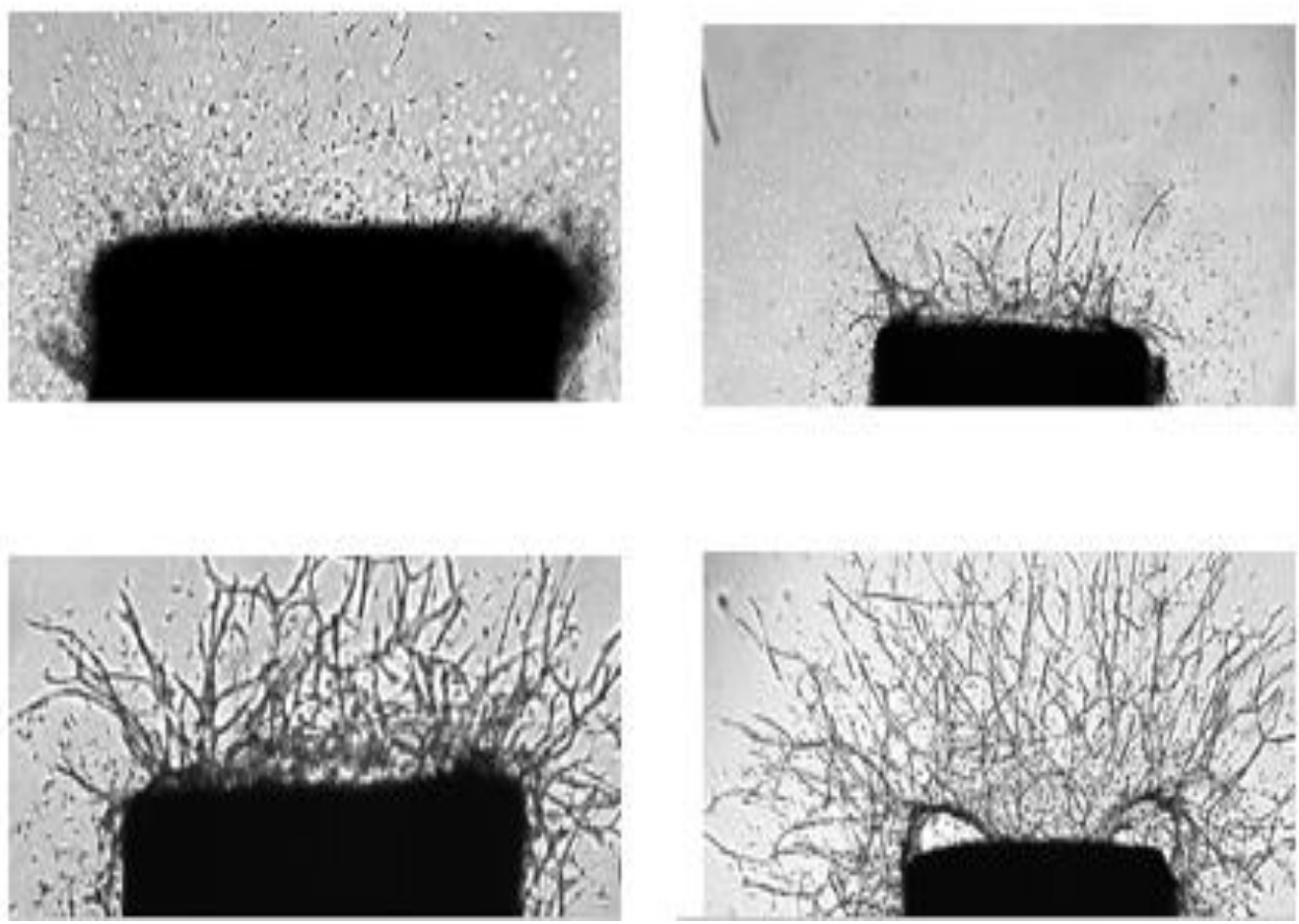

Figure 9 Effect of U94/REP on microvessel outgrowth in vitro. Chick aortic rings were embedded in type I collagen gels and incubated for 7 days ${ }^{22,23}$.

Standard imaging techniques are useful both for the enumeration of endothelial cells and for delineating the total outgrowth area.

The below table illustrates a brief idea on the advantages and limitations of various in vivo devices available in market 
Table 1 Advantages and limitations of various in vivo assays

\begin{tabular}{|c|c|c|}
\hline Assay & Advantages & Limitations \\
\hline CAM & 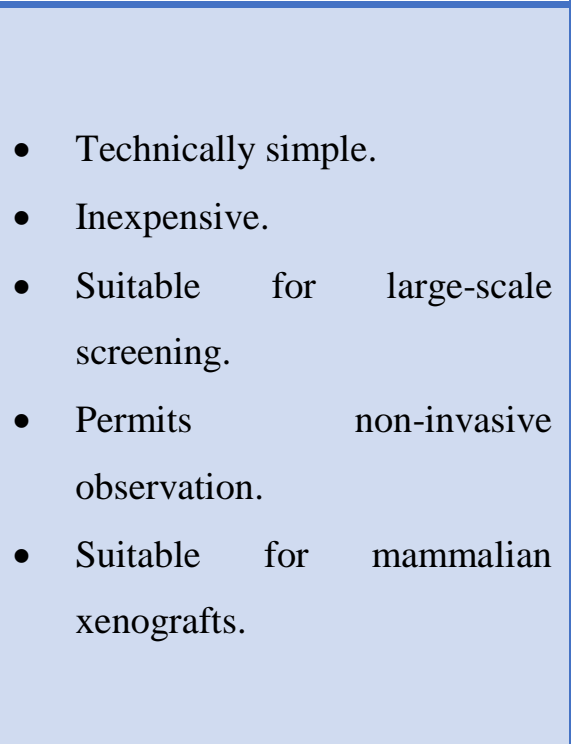 & $\begin{array}{l}\text { - Very sensitive to increase in oxygen tension. } \\
\text { - Visualization of new vessels can be difficult. } \\
\text { - Non-mammalian. } \\
\text { - Embryonic. } \\
\text { - Angiogenesis by sprouting and intussusceptive } \\
\text { growth. } \\
\text { - Accelerates or suppresses organogenic } \\
\text { angiogenesis (up to Days } 10-11 \text { ). } \\
\text { - Non-specific inflammatory reactions common. } \\
\text { - Drugs that require metabolic activation cannot be } \\
\text { assessed. }\end{array}$ \\
\hline $\begin{array}{c}\text { Sponge } \\
\text { Assay }\end{array}$ & $\begin{array}{l}\text { - Technically simple. } \\
\text { - Inexpensive. } \\
\text { - Well tolerated. } \\
\text { - Time course of response can } \\
\text { be recorded. } \\
\text { - Suitable for study of tumor } \\
\text { angiogenesis. }\end{array}$ & $\begin{array}{l}\text { - } \text { Time consuming. } \\
\text { - Encapsulated by granulation tissue. } \\
\text { - Sponge composition varies, making inter- } \\
\text { experimental. } \\
\text { - Comparisons difficult. } \\
\text { - Variable retention of test compound within } \\
\text { implant. } \\
\text { - The subcutaneous tissue is not a highly relevant } \\
\text { site for tumor growth. } \\
\text { - Animals have to be kept singly. }\end{array}$ \\
\hline DAS & $\begin{array}{l}\text { - Technically simple. } \\
\text { - Assesses wound healing and } \\
\text { angiogenesis. } \\
\text { - Quantitative analysis. }\end{array}$ & $\begin{array}{l}\text { - Encapsulated by granulation tissue. } \\
\text { - The subcutaneous tissue is not highly relevant for } \\
\text { tumor growth. }\end{array}$ \\
\hline
\end{tabular}




\subsection{Microfluidic Approaches}

Even though many in vivo and in vitro assays are available in market, a 3D analysis quantify and permit controlled perturbation of local cellular morphogenesis in a 3D matrix while also allowing for chemo kinetic and chemotactic effects.

Microfluidic technologies laid new way in monitoring cells which closely mimic in vivo conditions. The major advantage of microfluidic devices is, their abilities to provide adequate results in using very small quantities of reagents, stable gradient profile and its mimics the real time environment. Also it is capable to facilitate 3D cell migration and angiogenesis. The appropriate integration of engineering strategies and microfluidics has the potential to overcome the limitation faced by conventional approaches for the study of cancer.

\subsubsection{The angiogenetic sprouting of endothelial cells in 3D collagen gel matrix.}

Using microfluidics platform an in vivo like 3D angiogenesis environment was created in order to visualize and analyze the cell sprouting into collagen. In this thesis the angiogenesis tendency of four different EC were studied i.e. human umbilical vein endothelial cell (hUVEC), a human microvascular endothelial cell (hMVEC), a human aortic endothelial cell (hAEC), and

an endothelial colony forming cell (ECFC) ${ }^{12}$. Later the different tendencies of angiogenesis in each EC group are studied. Figure below shows the 3D model for angiogenesis study, with their channels and few supporting channels to load the chemo attractant.

Final concentration of the collagen solution $2.0 \mathrm{mg} / \mathrm{ml}$ was injected into the device, and the solution gelated in $37^{\circ} \mathrm{C}$ incubator for $30 \mathrm{~min}$. Later the corresponding EC's were seeded into the micro channels. 

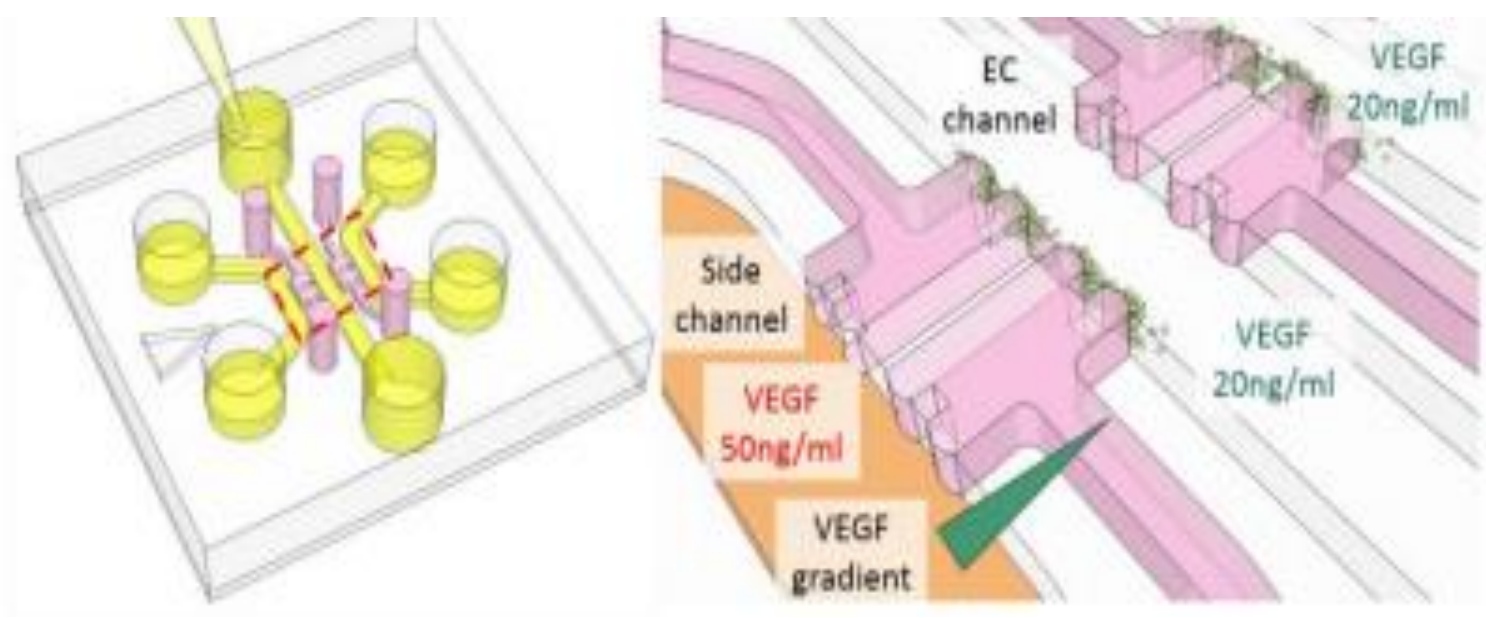

Figure $103 \mathrm{D}$ in vitro model for HUVEC cell angiogenesis

For inducing the angiogenesis, they generated a vascular endothelial growth factor (VEGF) gradient in the microfluidics platform during all the cultures. Growth media supplemented with different concentrations of VEGF (EC channel: $20 \mathrm{ng} / \mathrm{ml}$ and side channels: 50ng/ml) were added into the micro channels. Media were changed every 24 hours. ECs angiogenesis tendency were measured by gel penetration region and sprout capillary like region.

After determining the angiogenesis behavior of all EC's it is concluded that each EC's have significant migration behavior, and also collagen concentration do have effect in cell proliferation and angiogenesis behavior.

\subsubsection{In vitro based 3D angiogenesis sprouting}

This approach reports the engineering of $3 \mathrm{D}$ capillary beds in an in vitro microfluidic platform that is comprised of a biocompatible collagen I. This device has the capability coculture cells that either promote angiogenesis or require perfusion for cell viability in engineered tissue constructs. 

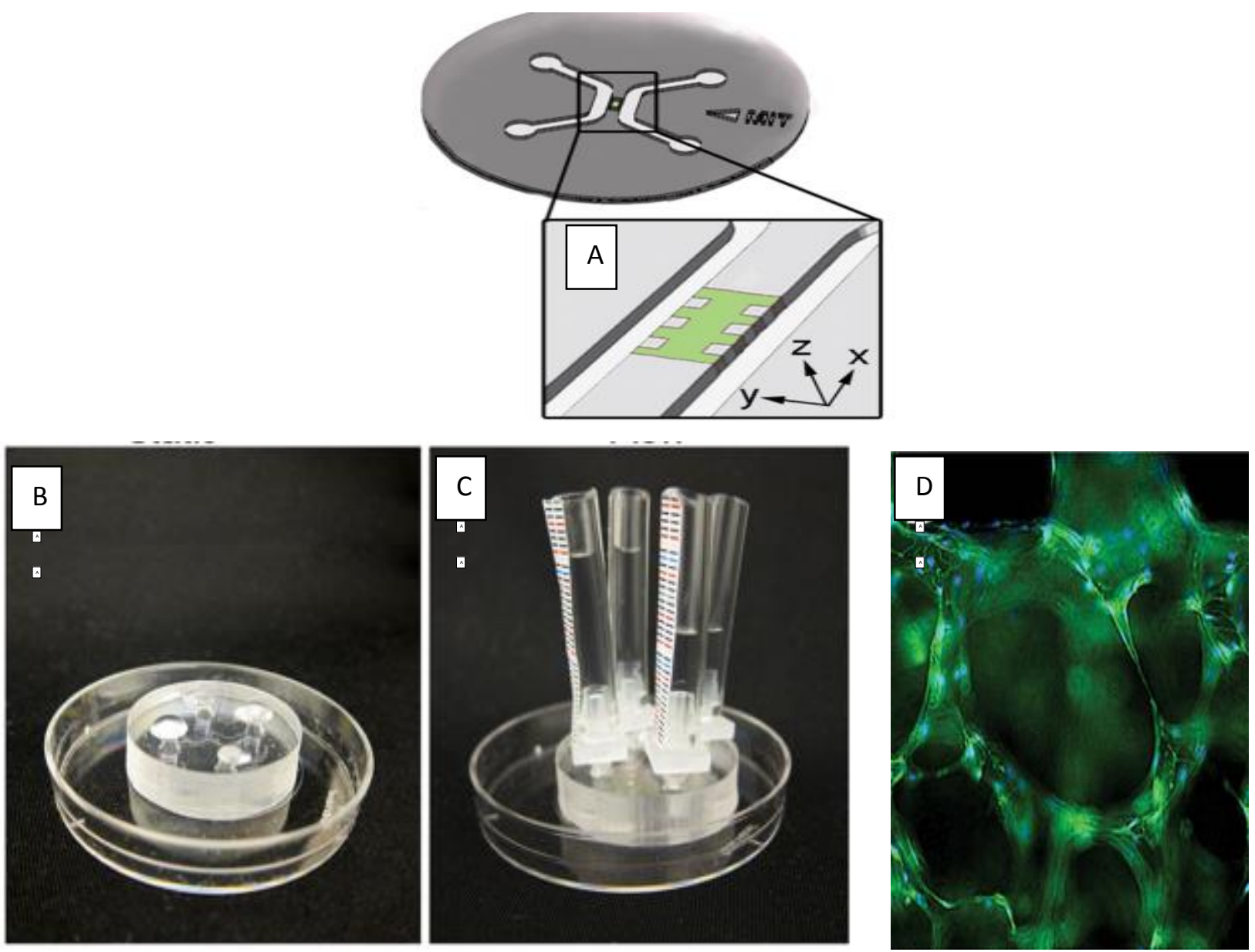

Figure 11 A) The microfluidic-based cell culture platform directs vascular growth along an alginate bead scaffold to form a 3D capillary bed; B), C) Microfluidic devices cultured under static and flow conditions D) Growth of HUVEC cells sprouting after 3 days growth ${ }^{13}$

\subsubsection{Current Microfluidic Assay}

After vigorous study of different in vivo microfluidics devices and their use in cell angiogenesis, a new microfluidic design is created in order to eliminate fewer limitations faced by the above microstructures.

- Complex in design.

- Harder to handle micropipette based filling.

- Uses of most studied EC's and analyze tubing formation in more simple and clear way. 
The motto of the microfluidic assay which is used in this thesis study considers to minimize all the possible limitations faced in prior microfluidic angiogenesis analysis experiments and came up with a new, simple and easy to handle model, which still serves the purpose like: linear chemotaxis gradient and detecting tubing formed by angiogenesis sprouting. 


\section{CHAPTER 3: MICROFLUIDIC 3D GRADIENT ASSAY}

\section{DESIGN}

\subsection{Device Layout}

AutoCAD is a widely-available computer-aided design package that is very useful for engineering layout of geometric features, including a high degree of detail, complexity, organization, and replication. These aspects make it a very convenient tool for low-cost photolithography mask design. ${ }^{28}$ A low-cost alternative to making traditional chromium-based photolithography masks is printing onto transparent plastic film using high-resolution graphics which is shown in figure 12 shows the mask layout design using AutoCAD and the manufactured chrome photomask.
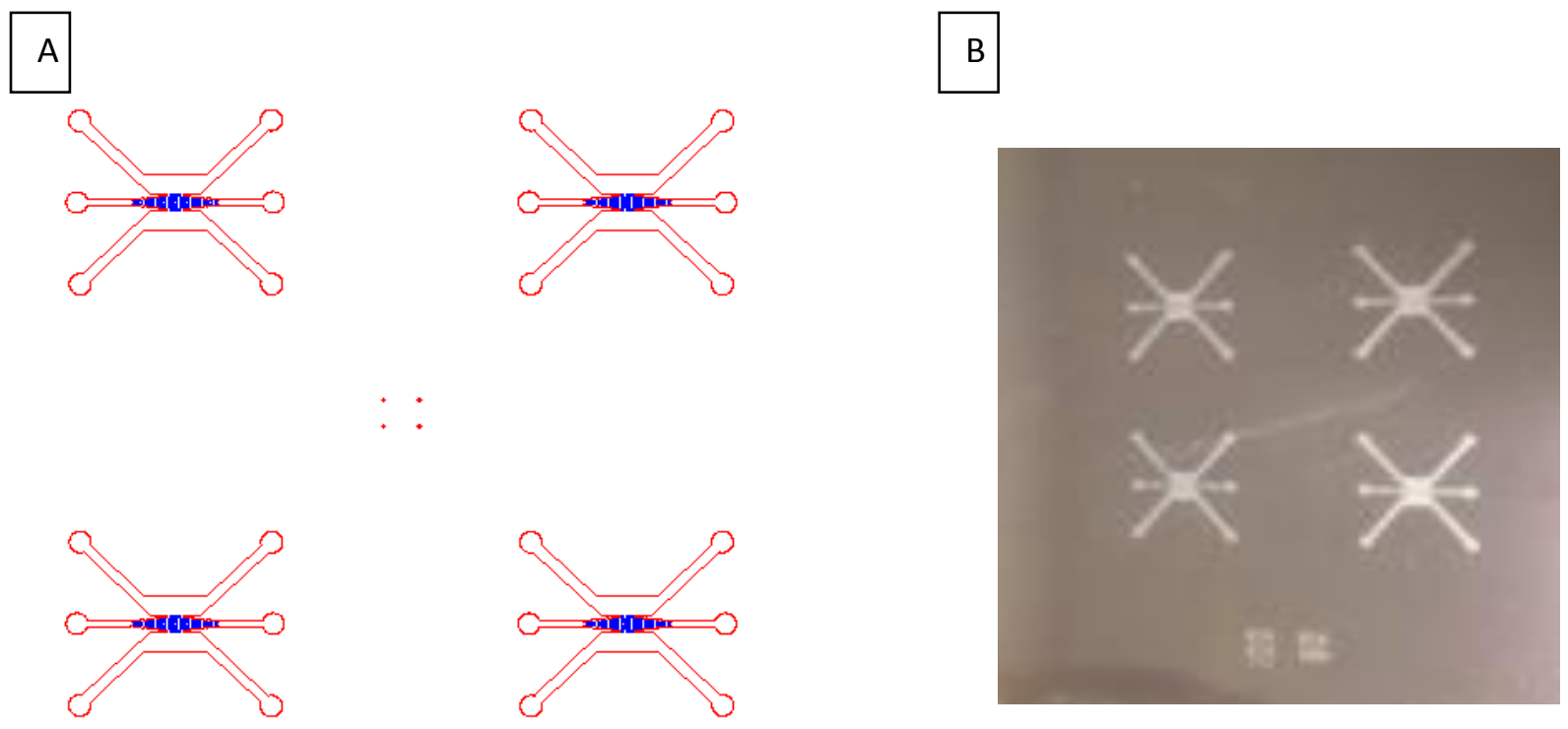

Figure 12 A) Microfluidic photomask layout using AutoCAD and B) Photomask 


\subsection{Microfluidic Gradient Device Design and Specifications}

\subsubsection{Initial Sketch}

The designed patten has 3 inlets and 3 outlets. The specifications along with the layout is as shown in the figure. Each inlet and outlet corresponds to one channel. All the inlets and oulets are designed with same diameter of $1000 \mu \mathrm{m}$. Either top or bottom channel is used for loading cells with medium on one side and chemoattractant on other side. The middle channel is filled with collagen. The choice for going to the 3 channel device (i.e having 3 inputs and 3 outputs ) layout is for better handling of all the loaded solvents. In order to mimic the real time system, an ECM (Collagen I) need to be sandwiched between the cell loading chamber and chemo attractant chamber. Hence by considering all the points, a 3 chamber layout is preferred in this thesis.

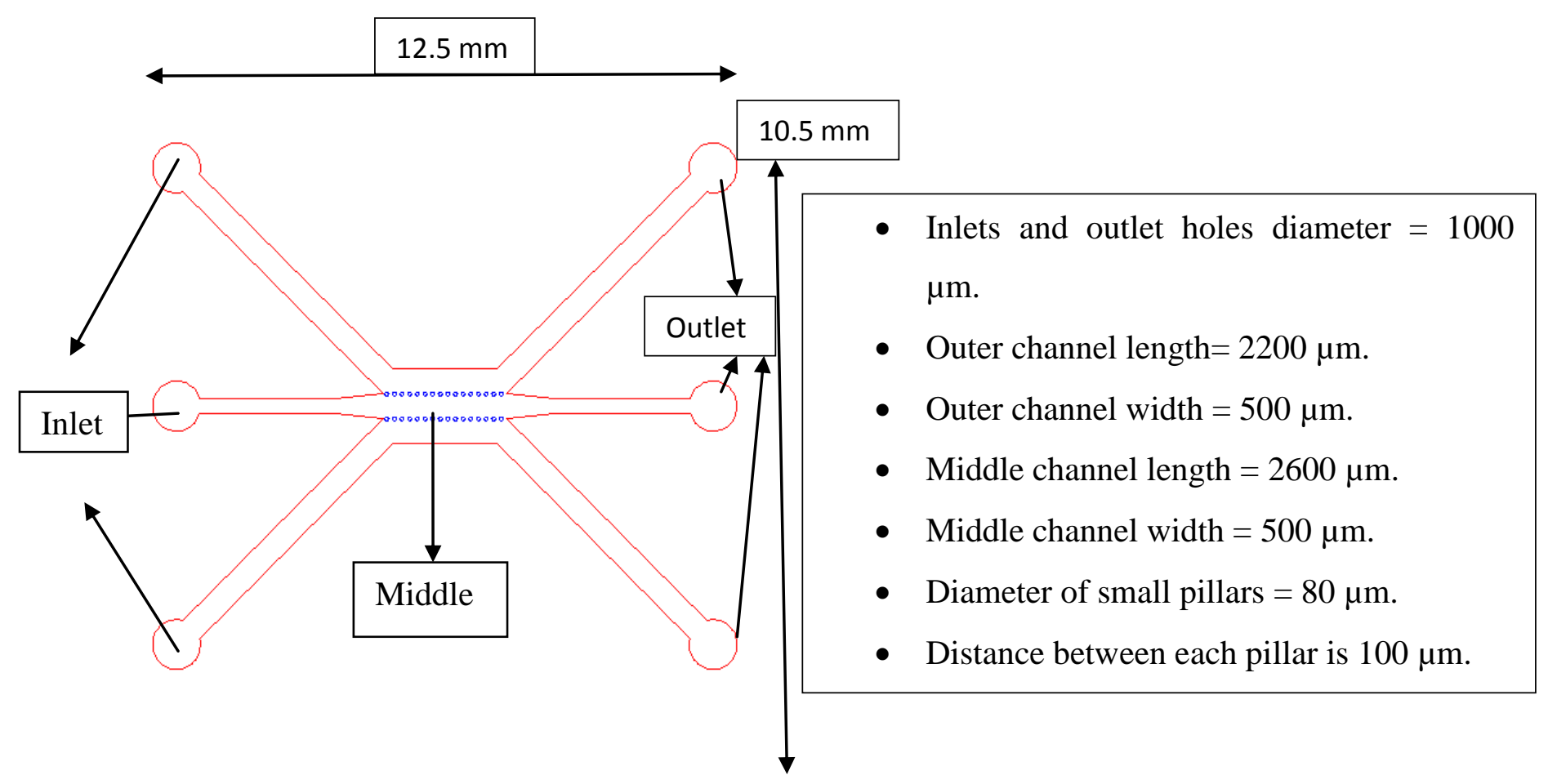

Figure 13 AutoCAD layout and specifications of a 3D gradient device. 


\subsubsection{Limitations and Modifications}

Breakage of collagen was widely observed by using this model i.e. the collagen is observed in top and bottom channels, which indeed is blocking the fluid flow in those channels. Secondly the gradient is not uniform as the collagen layer is not good enough to hold.

To minimize this effect, an array of pillars were introduced in the middel channel so that the collagen fibres will stick to them and better control of gradient can be seen. Apart from that, the curvy surfaces were introduced in the middlechannel for good collagen layer formation.

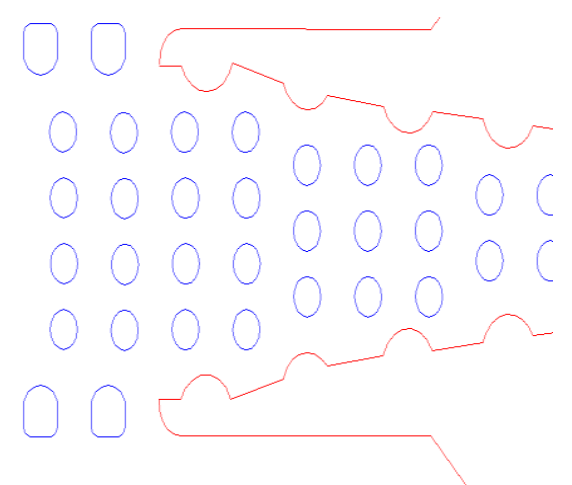

Figure 14 AUTOCAD model with curvy surfaces and pillars

Later for the double layer fabrication, new layer with extra wide top and bottom channels were introduced (larger surface area). The reason for this extra modification is to minimize the medium dry out and provide enough medium for the cells to survive. 


\subsection{Current Model for the Study of Tubulogenesis}

By analysing the drawbacks from the previous model,a new moodified design was implemented.This design includes 3 inlets and 3 outlets design.

Wide top and bottom channels with length and width of $2300 \mu \mathrm{m}$ and $950 \mu \mathrm{m}$ respectively and the middle channel width is modified to $800 \mu \mathrm{m}$ and has 4 openings each are of $100 \mu \mathrm{m}$ wide.Middle channel has an array of small pillars each of $80 \mu \mathrm{m}$ diameter and are seperated by $100 \mu \mathrm{m}$ distance. These small array of pillars are extended to some extend of the entrace and exit of middle channel. Curvy structures were also introduces in the middle channel which is shown in figure.

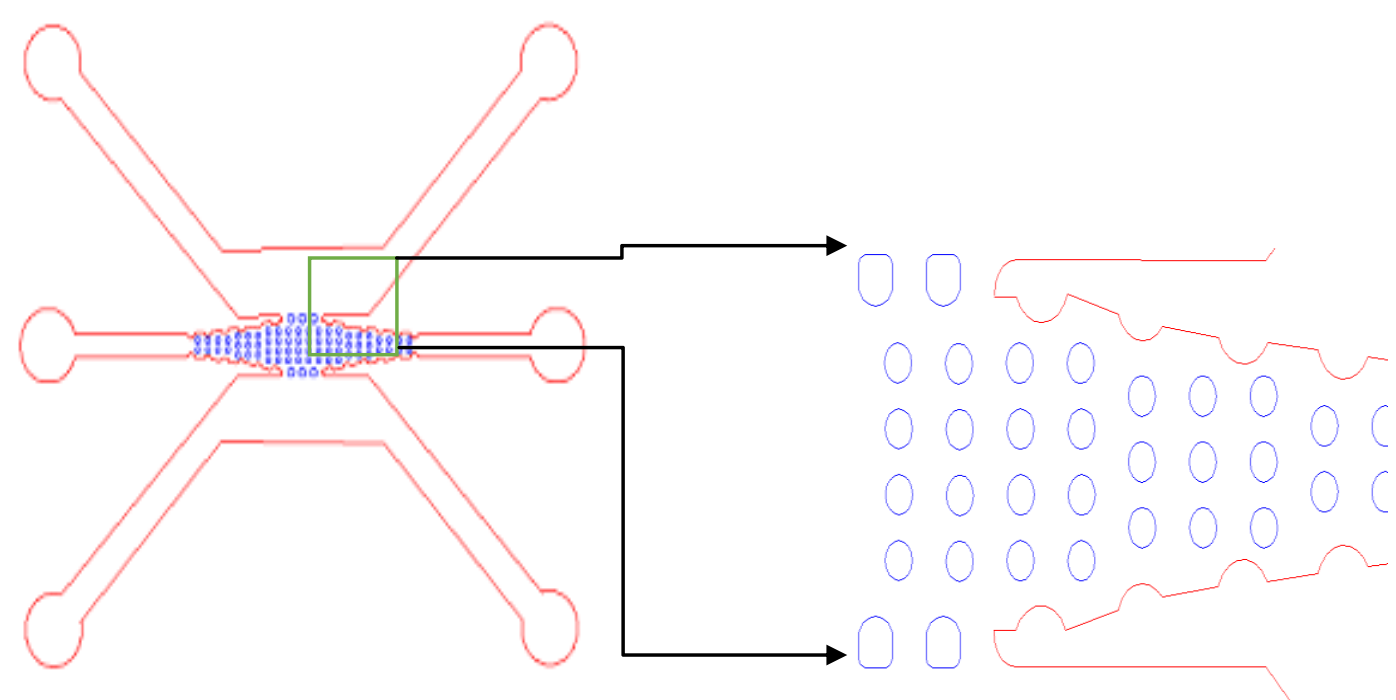

Figure 15 Modified AutoCAD design and Maximized image of the middle channel with pillars and curvy surfaces

Four of these designs are observed in each Wafer along with makers at the center. The need of markers is for correct alignment of the second layer. A chrome mask design of wide top and bottom channels is shown in figure 16 below. 

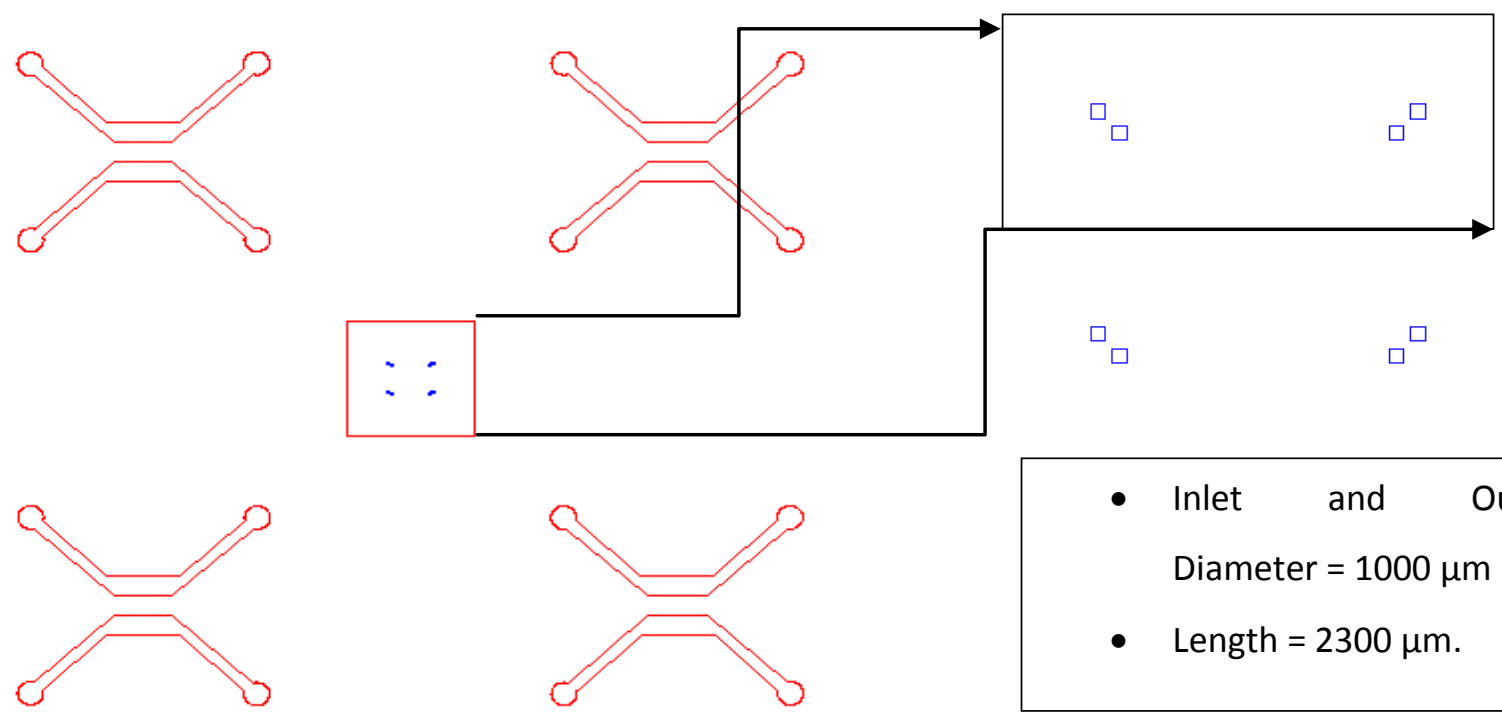

- Inlet and Outlet Diameter $=1000 \mu \mathrm{m}$

- Length $=2300 \mu \mathrm{m}$.

Figure 16 Double layer chrome mask layout 


\section{CHAPTER 4: MICROFLUIDIC CHIP FABRICATION}

The fabrication procedures are mostly the same in order to fabricate microfluidic chips regardless the design of the wafer. In brief the wafer is prepared, and a fluidic layer (generally PDMS) is deposited on the top. Clear idea of its fundamentals is described in detail below.

\subsection{Wafer Preparation}

Even though the wafer looks clean and tidy to our naked eye, it is supposed to make the patterned surface extremely clear from minute dust. So, the 3 inch ( $\mathrm{Si}$ - Wafer) which is used in this experiment must undergo cleaning process before depositing any fluidic layer. Wash the wafer with acetone for 5 minutes and dump the residue in the proper waste bottle and rinse the wafer with de-ionized water. Repeat the process with isopropyl alcohol for 5 minutes and dump the residue in the proper waste bottle. Blow dry with nitrogen gun to remove the moisture and dehydrate the wafer on the top of hot plate at $180^{\circ} \mathrm{C}$ for 30 minutes.

\subsection{Pattern Transferring}

Before transferring the designed pattern to Silicon substrate, the top surface need to be coated with thin layer of corresponding photoresist ( here SU-8 photoresist). In most of the fabrication process the photoresist coat is mandatory; resists may vary depending on the type of design and the expected outcome. In this thesis all the patterns used negative SU-8 photoresist, even for the case of double layer fabrication the same resist is used as a coat. 
Steps involved in this process are as follows:

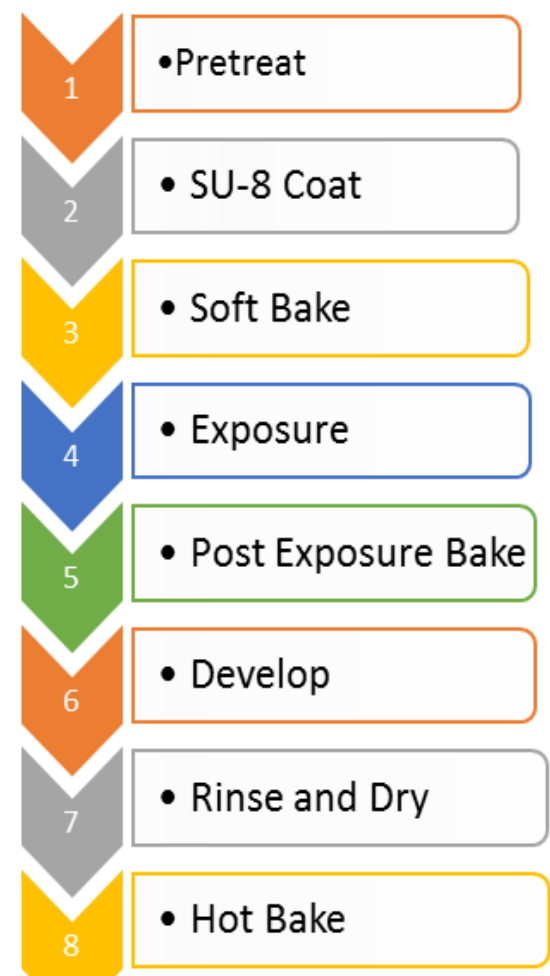

Figure 17 Steps involved in Lithography

1. Pretreat: The process of wafer pretreatment is clearly described above.

2. SU-8 Coat: SU-8 is an epoxy based negative photoresist where the number represents it has 8 epoxy groups. It was first developed by IBM in early 1982.The main reason for using SU-8 is because of its high adhesion capability, near UV- sensitive and possesses high aspect ratio ( 15 for lines and 10 for trenches $)^{29}$. Because of these properties it is widely used in the areas of sensors, actuators, electroplating molds and in preparing micro to mille scale structures.

The SU-8 used in this experiment is SU-8 2075. The film thickness versus spin speed table is as shown in the table. 
Table 2 : SU8 2075 data of thickness and spin speed.

\begin{tabular}{|c|c|c|c|}
\hline Resist Name & $\begin{array}{c}\text { Viscosity } \\
(\mathbf{c S t})\end{array}$ & $\begin{array}{c}\text { Thickness } \\
(\mu \mathrm{m})\end{array}$ & $\begin{array}{c}\text { Spin speed } \\
(\mathbf{r p m})\end{array}$ \\
\hline SU-8 2075 & 22000 & 75 & 3000 \\
\hline & & 110 & 2000 \\
\hline & & 225 & 1000 \\
\hline
\end{tabular}

As we are using SU-8 207s the recommended coating conditions are:

- Static Dispense: Approximately 1ml of SU-8 2075 per inch of substrate diameter.

- Spread cycle: In order for the resist to cover the whole surface set a speed of $500 \mathrm{rpm}$ for $10 \mathrm{sec}$ at an acceleration of $100 \mathrm{rpm} / \mathrm{second}$.

- Spin cycle: Set the spin speed to $1700 \mathrm{rpm}$ for $30 \mathrm{sec}$ at an acceleration of $300 \mathrm{rpm} / \mathrm{sec}$.

Actually the thickness of the photoresist depends on the spin speed, as the thickness of 150 $\mu \mathrm{m}$ is needed for this experiment the above parameters are set to use.

\section{Soft Bake}

After the spinning is done, the substrate is soft baked to evaporate the solvent and to densify the film. The recommended heating times are:

\begin{tabular}{|c|c|c|}
\hline Thickness & $\mathbf{6 5}^{\circ} \mathbf{C}$ & $\mathbf{9 5}^{\circ} \mathbf{C}$ \\
\hline $150 \mu \mathrm{m}$ & 5 minutes & 30 minutes \\
\hline
\end{tabular}


For best results ramping and stepping of temperatures is recommended. Lower initial bake temperature makes the solvent to evaporate at most which results in better coating and better resist to substrate adhesion.

\section{UV Flood Exposure}

In general SU-8 optimizes for near UV(350-400nm).It is virtually transparent and insensitive above 400nm. For this experiment we need double layer fabrication, as it increases the total surface area, which helps the cells to be healthy longer and also for good control of collagen. So the first layer is fabricated using UV flood exposure and the second layer is fabricated using mask aligner.

\subsection{Single Layer SU-8 Fabrication using UV Flood Exposure}

After soft bake the wafer is ready for exposure. Meanwhile the initial step to do 15 minutes prior to the usage of UV flood exposure is to warm up the UV lamp. Turn on the meter and set it to constant intensity (CI) position. Measure the UV intensity using Karl Suss 1000 UV intensity meter and probe (figure 18).Start the process by opening the shutter by pressing the "LAMP TEST" button on the exposure timer. Record the UV meter value in $\mathrm{mW} / \mathrm{cm} 2$ Close the shutter by pressing the "RESET" button on the exposure timer. Once the reading is registered, the UV probe can be removed. 

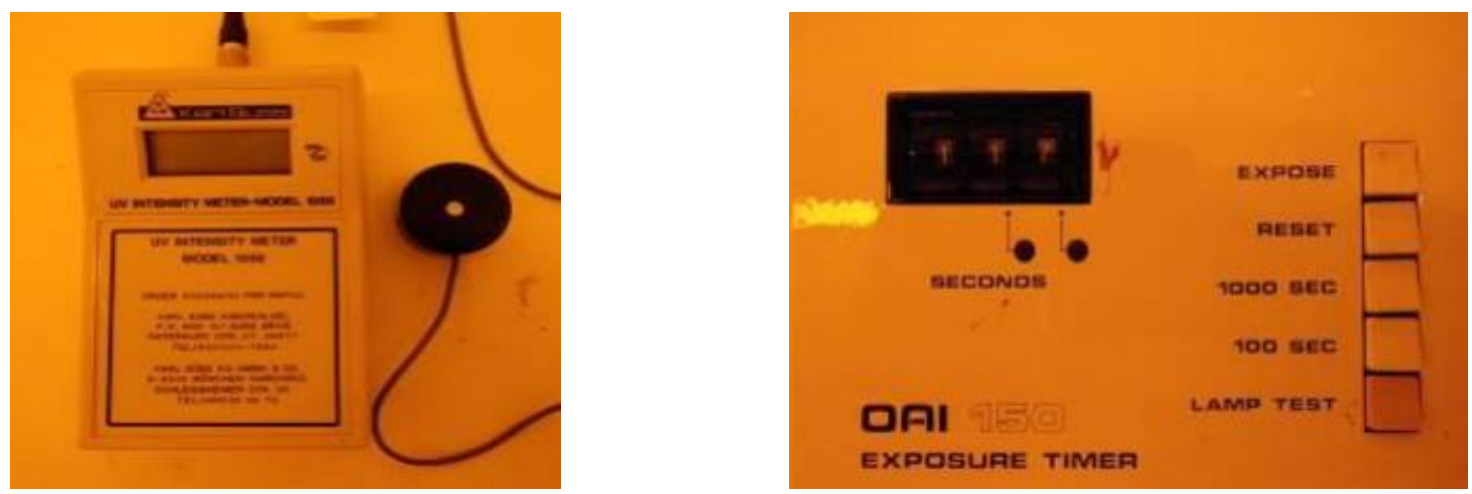

Figure 18 Karl Suss 1000 UV intensity meter and probe and Front panel of exposure timer

The recommended exposure energy for $150 \mu \mathrm{m}$ thick photoresist is $260 \mathrm{~mJ} / \mathrm{cm} 2$. Exposure time can be calculated as follows:

Time $=($ Recommended exposure energy $) /($ Recorded UV meter value $)$

Set the time by adjusting the second's buttons in the front plane and place the Si wafer where the pattern is supposed to be transferred under the Flood exposure system as shown in figure 19 .

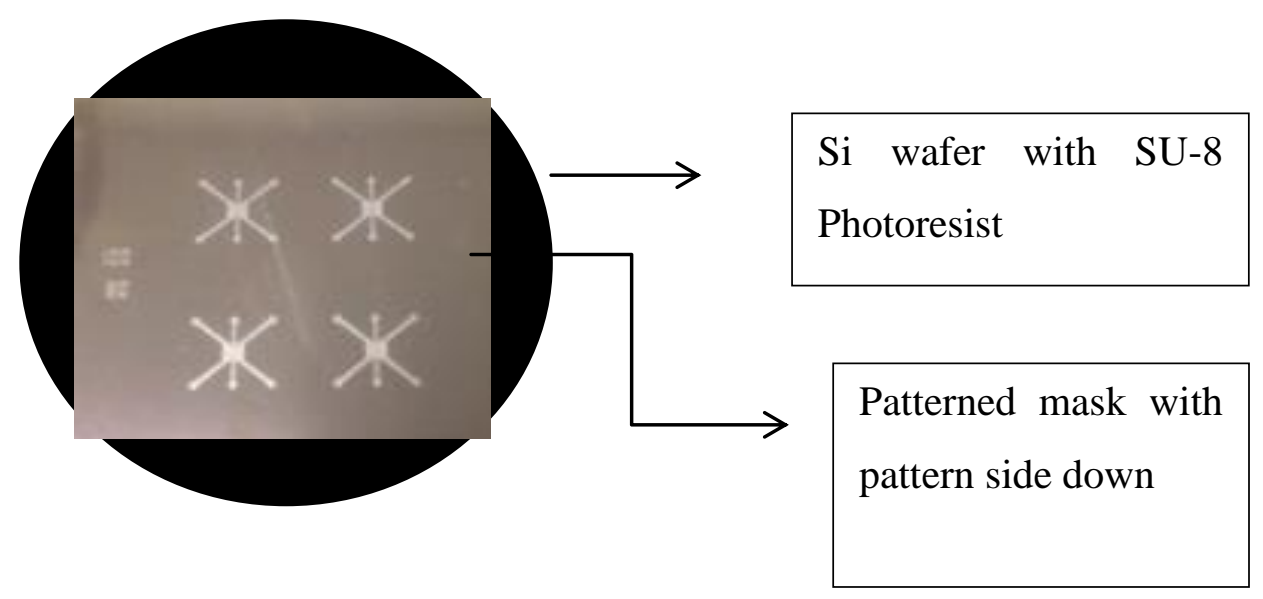

Figure 19 Wafer set up for UV exposure system

Press the Exposure button on the front panel, a pale violet light is observed. Once the UV is done the Si wafer is collected and set to Post exposure Bake. 


\subsection{Double Layer SU8 Fabrication using Mask Aligner}

In order to obtain the wide side channels, there is a need for double layer fabrication. Mask aligner is used for double layer fabrication using chrome mask (Made from very flat pieces of quartz or glass with a layer of chrome on one side). This step is done after the successful pattern transfer of single layer SU-8.

Most of the fabrication steps are in common with UV flood exposure system, the major difference is observed when the mask aligner operation comes into picture. To start the process power up the lamp controller by turning on the power switch. Once turned on, the lamp controller led panel will cycle through a warm-up procedure and then the display will read "rdy" for ready. Wait 15 minutes for the lamp to warm up. The led display on the lamp power supply should read 275 Watts. Turn on the vacuum pump and set the spinner speed to $500 \mathrm{rpm}$ for 10 seconds and 2100 rpm for 30 seconds.

Followed by soft bake for $5 \mathrm{~min}$ at $65^{\circ} \mathrm{C}$ and $20 \mathrm{~min}$ at $95^{\circ} \mathrm{C}$. Set the wafer to cool down, meanwhile measure the UV intensity using Karl Suss 1000 UV intensity meter and probe.

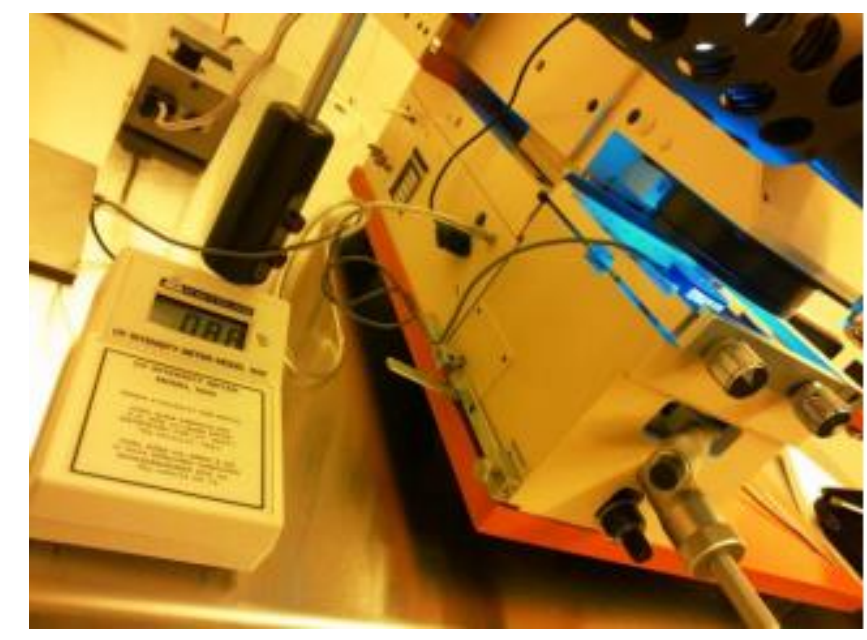

Figure 20 UV measurement using Karl Suss 1000 


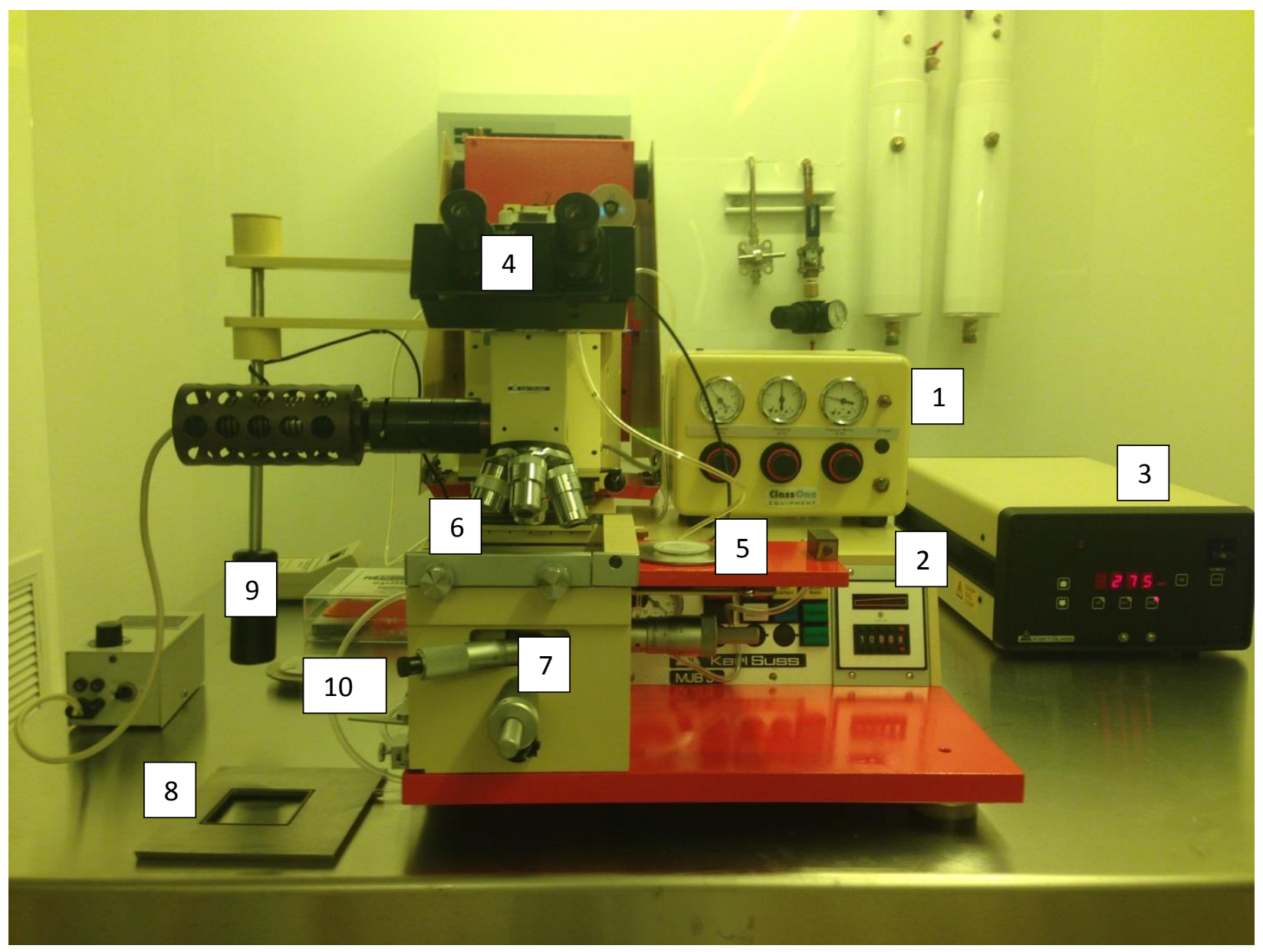

Figure 21 Mask Aligner (White hall clean room, WVU)

1. Nitrogen Pressures Reading

2. Control Panel

3. Lamp Controller

4. Microscope

5. Sample holder stage
6. Mask holder groves

7. XY and theta adjustment meters

8. Photo mask holder

9. Microscope Manipulator with X-Y Brakes

10. Contact Lever 
Once the exposure is done the exposure time can be calculated by dividing the desired exposure dosage, which should be in $\mathrm{mJ} / \mathrm{cm} 2$ by the UV intensity measured through the mask in $\mathrm{mW} / \mathrm{cm} 2$. Now Mount the photo-mask on the mask holder shown in Figure. The photo-mask should be positioned so that the chrome side of the photo-mask is facing up and manually centered over the mask holder opening. Place the sample on the sample holder and verify that all vacuum holes are covered. Adjust the position holders until better focus is observed from the microscope (using the $\mathrm{X}$ position, $\mathrm{Y}$ position, and $\theta$ position micrometers shown in Figure 21. Once the substrate is in contact with the photo-mask, set the timer to the desired set-point for exposure on the exposure time.

\section{Post Exposure Bake}

It is followed immediately after Exposure. Generally it is the most important step as it helps for the cross linking of the polymers. The image of the transferred pattern can be visible within 1-2 minutes of the exposure, if not the exposure is not enough. The recommended PEB time is as follows:

\begin{tabular}{|c|c|c|}
\hline Thickness & $\mathbf{6 5}^{\circ} \mathrm{C}$ & $\mathbf{9 5}{ }^{\circ} \mathrm{C}$ \\
\hline $150 \mu \mathrm{m}$ & 5 minutes & 12 minutes \\
\hline
\end{tabular}




\section{Development}

SU8 developer is used for the development of the SU-8 photoresist after exposure. Depending on the thickness and viscosity of the photoresist, development time varies. The recommended development time for $150 \mu \mathrm{m}$ thick photoresist is $12-15$ minutes. Actually the development process need to handle very gently as vigorous movements may leads to the wash away of patterns.

Couple of minutes of ultrasonic bath is also helpful sometimes. The best thing is to monitor the pattern after couple of minutes and then to proceed further.

\section{Rinse and Dry}

Once the required patterns are observed after development, the silicon mode is washed with DI water and then blow dry with nitrogen gas. Sometimes few residue of photoresist is observed, then a gentle wash with developer followed by DI water leaves a clear silicon mode with perfect visibility of pattern.

\section{Hot Bake}

SU-8 2000 has good mechanical properties. However, for applications where the imaged resist is to be left as part of the final device, a hard bake can be incorporated into the process ${ }^{30}$. A hard bake or final cure step is added to ensure that SU-8 2000 properties do not change in actual use. The hard bake step is also useful for annealing any surface cracks that may be evident after development. The recommended step is to bake at $150^{\circ} \mathrm{C}$ for like 20 minutes. This applies to all film thicknesses. 


\subsection{Potential Problems and Solutions}

Because of over development and high temperature: cracks are observed on the surface of the pattern, which can be minimized by taking care of development time. Sometimes the large patterns get washed away and the small features are not yet developed as shown in Figure (22) While developing, the smaller areas are taken care first as the space is very narrow it takes longer time to develop.

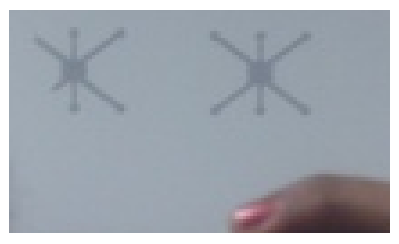

Figure 22 Pattern washed away due to over development

\subsection{Soft Lithography}

Soft lithography is a technology where replicating structures are formed using elastomeric stamps, molds, and conformable photomasks ${ }^{31}$. And the elastomeric material used is most notably Polydimethylsiloxane (PDMS) which is soft.

Generally fabrication of a PDMS layer requires three steps:

1. Patterning a mold master from SU-8,

2. Casting PDMS on the mold master

3. Removing the PDMS from the mold (shown in Figure 14) 

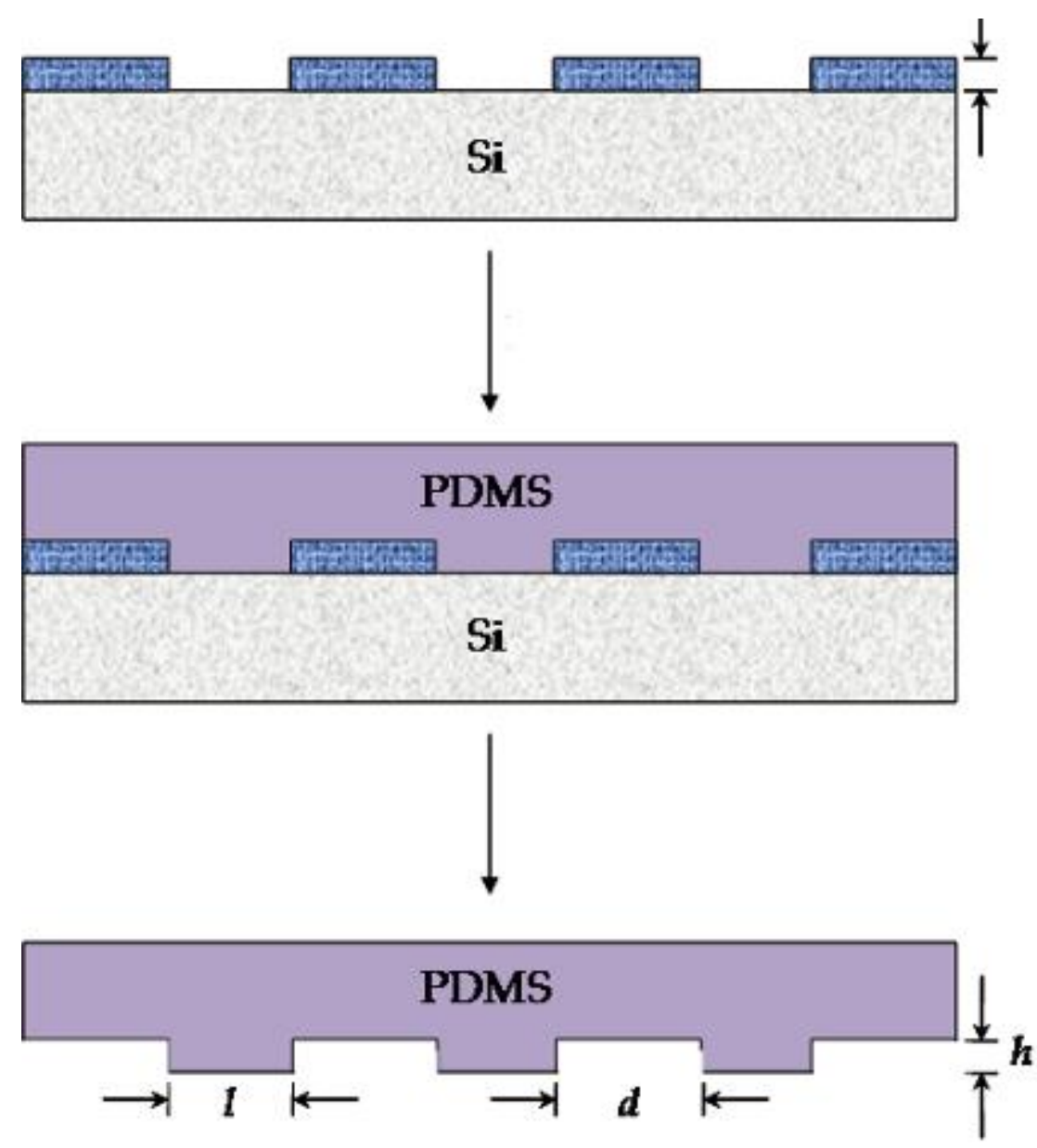

Figure 23 Schematic illustration of the procedure for casting PDMS replicas from a master having relief structures on its surface.

\subsubsection{SU-8 Master}

The SU-8 master mold is fabricated same as discussed above in SU-8 layer fabrication. The mold mask design is a negative: the negative resist remains on the surface wherever it is exposed, and the developer solution removes only the unexposed portions, i.e. same features in plain SU-8 - "hills" in the SU-8 are needed to create "valleys" in the molded PDMS. 


\subsubsection{PDMS Mode}

In order to create a layer of PDMS, the polymer is poured on the top of the appropriate mold and then cured in an incubator. Here are the steps to follow:

1. Mix PDMS base (Sylgard 184): Curing agent in the ratio of 10:1 and spin it at 2000 rom for 30 seconds.

2. Pour this solution on the top of the master mold and degas for 30 minutes.

3. Carefully remove the remaining air bubbles using nitrogen gun and place the device inside the incubator for 2-3 hours.

\subsubsection{Demolding PDMS}

Demolding PDMS is a delicate process and care must be taken in each step. After collecting the whole device from incubator and carefully loosen the surroundings by using scalpel.

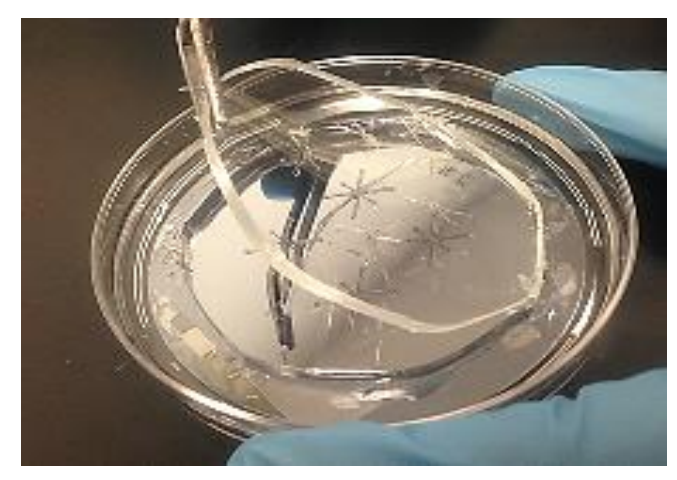

Figure 24 Demolding PDMS wafer from Master Mold

Remove the PDMS mold from the Master mold (Figure 24) and tape clean the surface to remove minute dust particles. Punch holes and place in plasma chamber and UV treat them for 
$30 \mathrm{sec}$ (Pattern side up).Carefully bond PDMS to glass which forms an irreversible bonding. Incubate for 15 minutes in order to have a strong non leakage bonding.

\subsection{Potential Problems and Solutions}

As PDMS processing is easy and multiple modes can be made from single master mode, the few difficulties faced are, Demolding PDMS from the mode. The demolding process is not complicated but it needs sufficient practice to handle the pattern without damaging it. And the other thing is loosen the PDMS using Scalpel may create rough edges, which may result in cracks in future.

The second problem is as the design includes small pillars of 80 micros, after multiple usage of the mode we can see that the pillars are blocked. So in order to get of this problem SIGMACOTE (Aldrich) is used. SIGMACOTE is a clear, colorless solution of a chlorinated organopolysiloxane in heptane.

\section{Application}

- SIGMACOTE reacts with surface silanol groups on glass to produce a neutral, hydrophobic, microscopically thin film. The film repels water, retards the clotting and helps to reuse the mode.
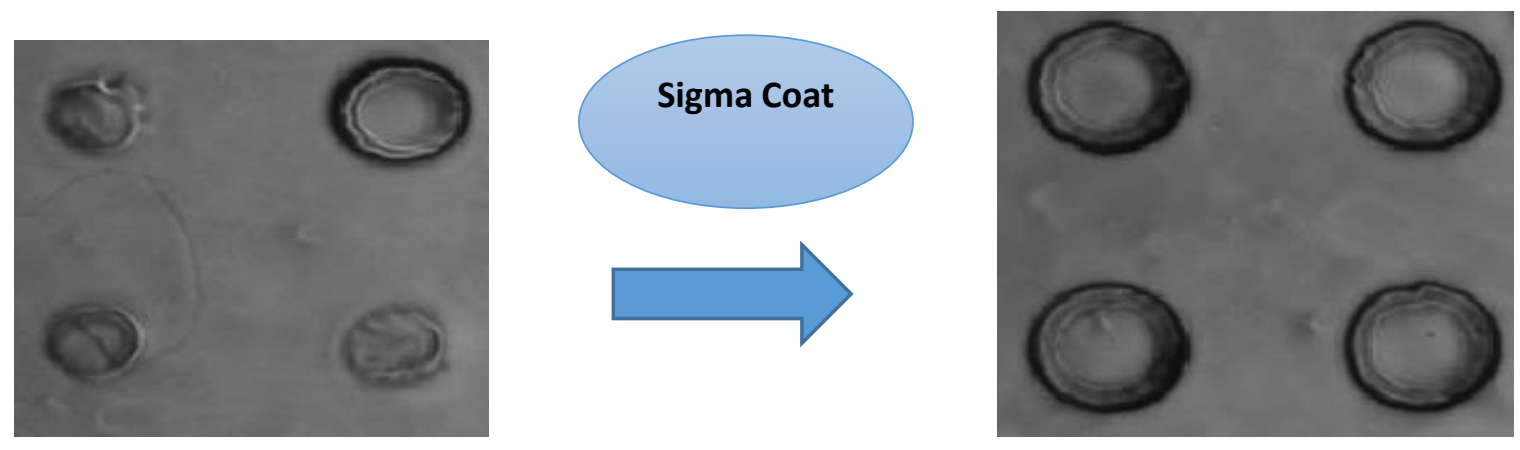

Figure 25 Blocked pillars due to PDMS and Clear pillars due to usage of Sigma Coat 


\section{CHAPTER 5: CELL CULTURE IN MICROFLUIDIC}

\section{DEVICES}

Culturing mammalian cells in in vitro environment is quite challenging as utmost care must be taken in creating a suitable and healthy environment. In this experiment Human umbilical vein endothelial cells (HUVEC) were used. HUVEC are cells derived from the endothelium of veins from the umbilical cord. They are used extensively in various lab application studies because they are economical compared to other primary cells.

Few studies in which HUVEC cells were used are: Cardiovascular pharmaceutical development, vascular pathology, Atherosclerosis, Angiogenesis Inflammation and Wound healing. In this experiment HUVEC are used in the study micro tube formation inside microfluidic device. The clear view of its culture is discussed below.

\subsection{Cell Culture}

Cell medium is a source of nutrient for the cells and thus is important for proliferation of mammalian and other cell lines. The cell medium used was MCBD 131 with L-glutamine and supplemented with $20 \%$ of fetal bovine serum (FBS). The medium is set to change in every alternate days. The cells at the passages between 2 and 5 were used for the experiment.

When the HUVEC were confluent, the cells were counted and harvested by first rinsing the cells with HEPES Buffered Saline Solution (HEPES-BSS), and then the cells were treated with Trypsin/EDTA and incubated for $2-6$ minutes at $37^{\circ} \mathrm{C}$. After the trypsinization was complete the 
trypsin/EDTA was neutralized with trypsin neutralizing solution, the cells were then suspended in 8\% Dextran MCDB 131 culture medium to increase the viscosity to aid in better cell seeding and attachment. Once the cells are ready the next step is to load into microfluidic devices.

\subsection{Experimental Procedure}

The whole experiment was a 3 day process. Various modifications were included until we get the expected outcome. In this chapter all the initial methods along with the modifications made were discussed.

After bonding PDMS devices to the glass slide, the devices are filled with DI water. In order to avoid dry out Place the opening holes are covered with PDMS. Later place the whole device in petri dish with a wet tissue inside, and wrap with a parafilm.

\section{Pre experimental procedure}

The whole PDMS bonded device must be placed inside the laminar hood and expose to overnight UV light ( $\sim 8$ hours). All the needles and tubing used also must be autoclaved and should be operated inside the laminar hood. In order for the consistent laminar flow tubes were inserted into the inlet and outlet holes. Prepare a 2 inch long Tygon larger OD tubing and 12 inch Cole Parmer smaller OD tubing. Insert the Cole Parmer tubing into the Tygon tube and the other end of Tygon tubing will be connected to the syringe needle. Insert the Cole Parmer into the

device gently. There should be no bubble inside the tube, or in the device, a Cole Parmer tubing was inserted into the device as output. 


\section{Collagen Loading}

Collagen serves as an Extra Cellular Matrix (ECM) hence collagen with concentration: 2.5 $\mathrm{mg} / \mathrm{ml}$, which is the EZ gel Collagen Type I $(5 \mathrm{mg} / \mathrm{mL})$ diluted with serum free (M199 MeT5A) media was used to load inside the microfluidic device. As collagen cures at room temperature, before starting the experiment all the sterilized tubing, needles, syringes, water, and diluted collagen are required inside the refrigerator for overnight and have them completely cooling down. Set the syringe pumps, ice pack, syringes, DI water inside the hood after spraying $70 \%$ ethanol.

As whole set up is done under cold temperature, i.e. using ice packs. place the device on the top of ice filled dish and the device is of the same height as of the syringe pump. Initially set water tubing's, at the flow rate of $1.5 \mu 1 / \mathrm{min}$ and insert the tubing's to the PDMS mold once the droplet is out. The amount of collagen required inorder to fill the whole middle channel was about 1.2 microliters. Carefully collect required amount of collagen and attach the tubing to one end of the syringe needle. Once the collagen comes out from the other end of the tubing, insert gently to the middle inlet.

Set the flow rate of collagen to be $0.8 \mu \mathrm{l} / \mathrm{min}$ and let it run for 20 minutes. Make sure that there is no leakage and no bubbles trapped in between while injection. After the specified time, slowly turn off the collagen first followed by water. Leave the device undisturbed in $37^{\circ} \mathrm{C}$ incubator for 2 hours to get collagen cured. Wrap the device with parafilm to prevent evaporation. 


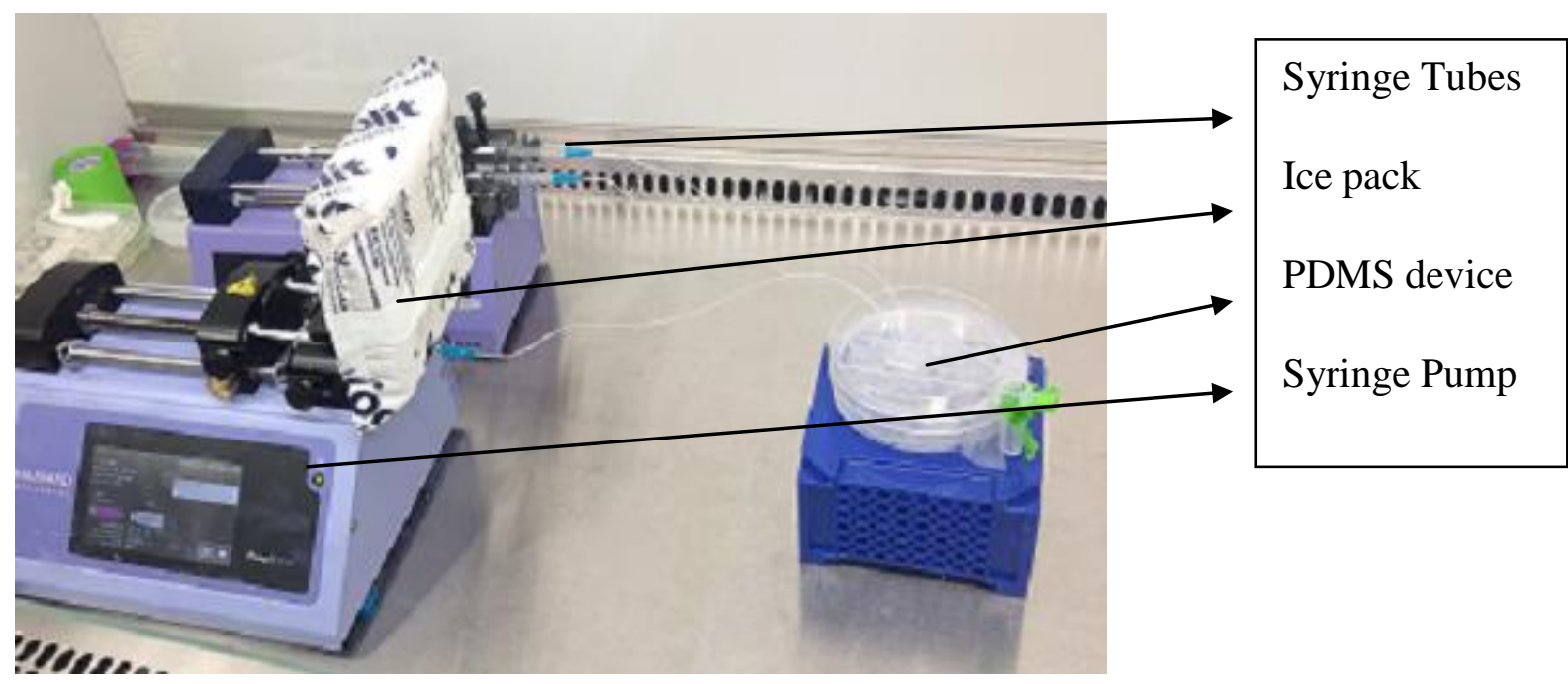

Figure 25 Experimental set up for collagen loading using syringe pumps

\section{Fibronectin Coating}

Collect the device and check under microscope in order to confirm the collagen level. Later using sterilized 1XPBS wash top and bottom channel of the device. Use pippet to load $20 \mu \mathrm{L}$ of PBS on the inlet, make sure it flush through the channel completely.

Note: Be very gentle as the excess force results in collagen breakage.

Coat the top and bottom channels with human fibronection (FN) (50 $\mu \mathrm{g} / \mathrm{ml}$ diluted with sterilized 1X PBS) for better attachment of cells. Make sure enough volume of FN solution loaded inside the top and bottom channels. $20 \mu \mathrm{L}$ per droplet of $\mathrm{FN}$ at the inlet, 6 times loading should be efficient. Cover the top holes with PDMS to prevent dry out and Wrap the device and leave it inside the $4^{\circ} \mathrm{C}$ refrigerator for overnight. Before loading the cells wash the top and bottom channels with serum free media. 
Once the media is observed form the other outlet, it is confirmed that the path (top and bottom channels are open for solution and cells flowing through) is made for the cells to reach the outlet. Make sure to warm up the device inside the $37^{\circ} \mathrm{C}$ incubator over 15 min before loading cells Now load cells very gently from one of the inlets and check under the microscope. Make a note of number of cells attached inside the channel and the path followed by cells. Make use of both the inlets of the corresponding channel to load cells if necessary. Load the certain concentration of FBS, i.e. $10 \%$, on the other channel. Later gently set up the whole device under microscope and take time lapse images overnight. Once the gradient is formed, we can observe the cells invaded into the middle collagen layer and towards up to the gradient direction to the other channel. Capture images in each stage.

\subsection{Potential Problems and Solutions}

Leakage: The main reason for the leakage of fluids is due to its poor bonding. In order to minimize this as soon as the plasma treatment of both the surfaces is done they must bring together for bonding. And also place the device inside the incubator for 15 minutes, for better bonding.

Trapping of air bubbles: This is the most common problem, as the device dimensions are so small it is very hard to remove if any air bubbles are trapped inside. The best solution is while handling fluids and tubing we need to make sure that they are bubble free. Before inserting the tubing to the channel inputs allow some liquid drops comes out to make sure the tubing is free from air trapping. 


\subsection{Modified Experimental Procedure}

From the above process, the most tedious and time consuming process is collagen loading. So later on, a new less tiresome process for collagen loading has been used and it was successful. The procedure is discussed clearly below:

Here there is no need of using tubing and syringe pumps for collagen loading which nearly reduces all the time. The devices are placed in laminar hood for overnight UV.

\subsubsection{PDL Coating and Collagen Loading}

Fill the channel surface with surface coating solution like PDL, which can prevent the detachment of collagen gel, resulting in 3D capillary morphogenesis. Place the devices in incubator for more 5-7 hours at $80^{\circ} \mathrm{C}$ followed by DI water wash for 5-6 times. Place the devices again inside the incubator at $80^{\circ} \mathrm{C}$ for more than 24 hours but less than 72 hours. It takes over 24 hours to restore hydrophobicity of the bonded surfaces.

Collagen with concentration: $2.5 \mathrm{mg} / \mathrm{ml}$, which is the EZ gel Collagen Type I $(5 \mathrm{mg} / \mathrm{mL})$ diluted with 10x PBS. Calculate the exact volume of middle channel and fill the collagen accordingly. (Here $1.6 \mu \mathrm{l}$ of collagen is sufficient to fill the whole middle channel).Now carefully insert the puppet tip into the middle channel and gently release collagen. Later place the device inside incubator for 1 hour at $37^{\circ} \mathrm{C}$ temperature. Coat the top and bottom channels with human fibronection (FN) $(100 \mu \mathrm{g} / \mathrm{ml}$ diluted with sterilized 1X PBS) for better attachment of cells. Make sure enough volume of FN solution loaded inside the top and bottom channels. $20 \mu \mathrm{L}$ per droplet of FN at the inlet, 6 times loading should be efficient. . 
Leave the system undisturbed for 2 hours and wash the top and bottom channels with 10x PBS as excess amount of FN may effect cells and later fill with HUVEC cell medium. Place the devices in the incubator for warm up before loading the cells. The cells used in this experiment id HUVEC cells and once the required confluence is observed they can be used to load on the devices.

The cells were counted and harvested by first rinsing the cells with HEPES Buffered Saline Solution (HEPES-BSS), and then the cells were treated with Trypsin/EDTA and incubated for 26 minutes at $37^{\circ} \mathrm{C}$. After the trypsinization was complete the trypsin/EDTA was neutralized with trypsin neutralizing solution, the cells were then suspended in 8\% Dextran MCDB 131 culture medium to increase the viscosity to aid in better cell seeding and attachment. The HUVEC cells in 8\% Dextran MCDB 131 culture medium were then loaded into the device with a cell concentration of $2 \times 10^{6} \sim 3 \times 10^{6}$ cells $/ \mathrm{mL}$. A $20 \mu \mathrm{L}$ droplet of cells was placed at one inlet and the device was tilted to introduce the cells into the microfluidic channel.

After 15-20 minutes cells began to attach to the sidewalls of the channels. The device was then rotated every 15 minutes to create a more uniform distribution of cells. If necessary, additional loading could be performed from the other inlet. Later on fill the channels with new medium and place it in the incubator overnight. Next aspirate the medium form the two inlets and make sure to fill the other side of the channel (which has no cells) with chemo attractant. VEGF is used as a chemo attractant for HUVEC cells, load the channel with $50 \mathrm{ng}$ ml - VEGF to generate a gradient across the gel scaffold. Culture the cells for a further 2-3 days by replacing the mediums twice daily. 


\subsection{Cell Staining}

When the cells reached confluence inside the device, firstly, the device was washed with PBS to thoroughly remove the medium. Fix the cells by diluting (500 $\mu 1$ of PBS with $50 \mu$ l of fixing solution- paraformaldehyde), place the devices in ice for 20 minutes. Wash them with PBS and then the device was loaded with TRITON X100 diluted with PBS (1 $\mu 1: 1 \mathrm{ml})$. The loading method was similar to the cell loading procedure.

The device was incubated in the dark for 3 minutes at room temperature. Then the device was washed with PBS. Next Step is Actin staining, dilute action with PBS in the ratio of (1:100) and then load the device with this solution. After incubation in the dark for 10 minutes at room temperature, the device was thoroughly washed with PBS. NucBlue Fixed cell stain by molecular probes is used to fix the nucleus, diluted with PBS (2 droplets per mL) was loaded into the device. After incubation in the dark for 5 minutes at room temperature, the device was thoroughly washed with PBS.

The cell staining was examined completely covered with aluminum foil and stored at $4^{\circ} \mathrm{C}$ in the refrigerator to prevent the device from drying out and bleaching. The fixed device was ready for confocal imaging. 


\section{CHAPTER 6: RESULTS AND DISCUSSION}

\subsection{Gradient Analysis}

In this approach, Collagen type I is loaded in the gel region of the microfluidic device to achieve physiological relevance, in turn promoting 3D angiogenesis under well-controlled microenvironments. ${ }^{32}$ In addition, HUVEC cells were loaded for cell culture in microfluidic devices and formation of tubogenesis. Cells were cultured in one channel in direct contact with collagen scaffolds. Once a confluent endothelial monolayer is formed, induction of an in vivolike angiogenesis is observed.

After loading Collagen I into the middle channel of the device, the device is placed at $37^{\circ} \mathrm{C}$ for 1 hour. The analysis of the collagen bonding formation was visualized and quantified with the study of gradient profile using Fluorescein isothiocyanate (FITC)-albumin (Sigma-Aldrich, Saint Louis, MO). 10\% FITC solution was gently loaded into one of the inlets without damaging the collagen bonding. Fluorescence images were taken for every 15 minutes for 20 hours using an inverted microscope (Nikon Eclipse Ti, Japan). Every recorded value of the fluorescence intensities were analyzed using Sigma plot software. Figure 1 shows the formation of gradient across the middle channel (collagen channel), where FITC is loaded at the top channel. Slow and steady based diffusion based gradient profile can be clearly seen across the channel after the end of 4-5 hours. Figure 26 clearly indicates the gradient profile of a stable gradient with normalized intensities across various time periods. The gradient in the migration channels was generated mainly by diffusion $\left[{ }^{33}\right]$. 


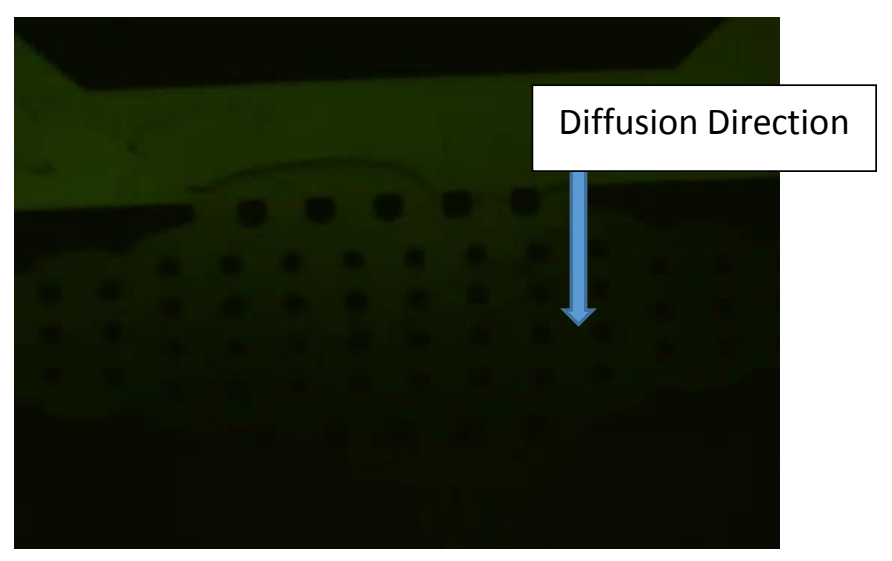

Figure 26 Fluorescence image of generated gradient across the collagen channel.

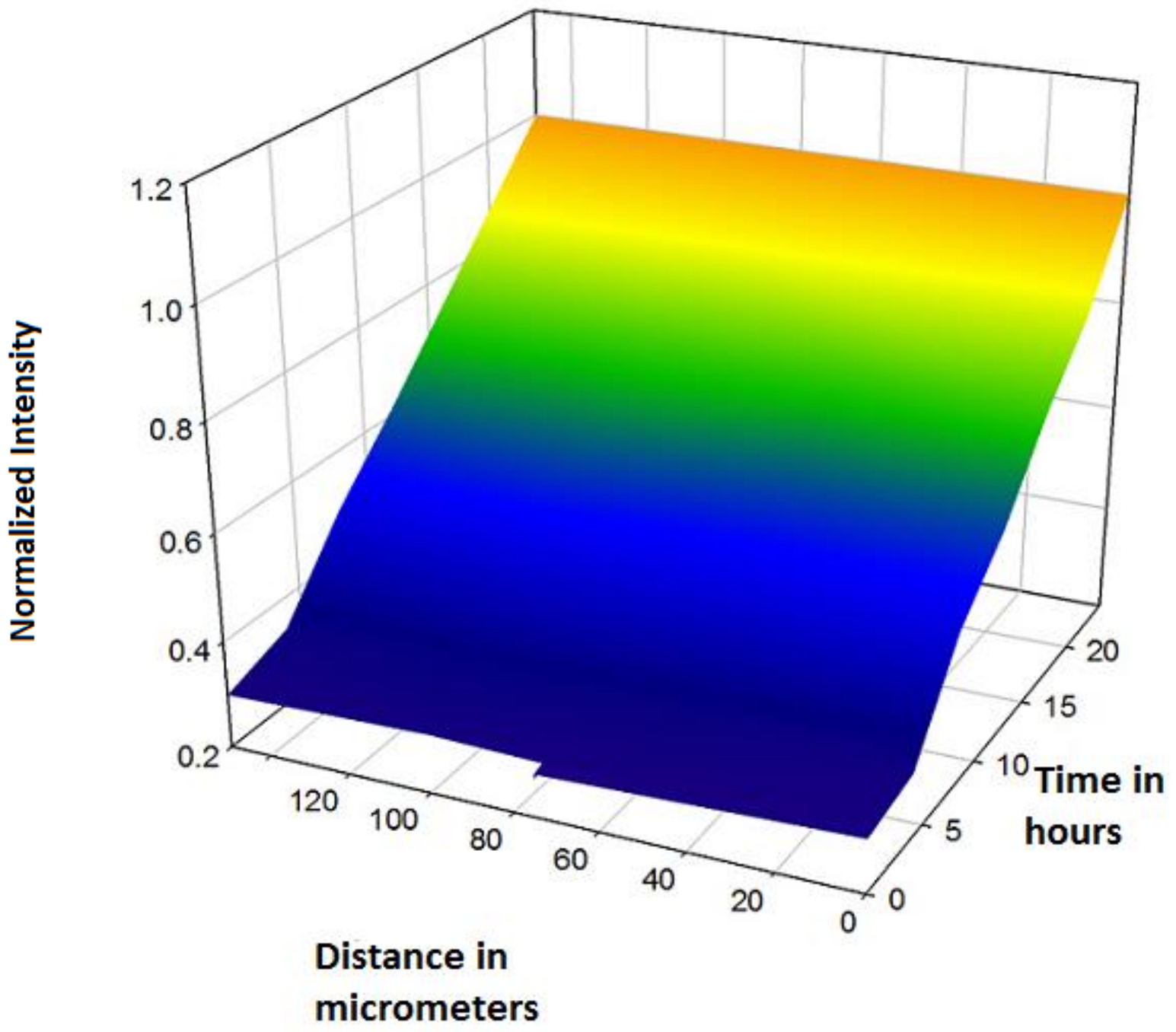

Figure 27 FITC-albumin gradient across the width of the channel, a stable linear gradient through the middle collagen channel. 
As $3 \mathrm{mg} / \mathrm{ml}$ of collagen is loaded, there are cases of mild distraction in collagen bonding while loading other liquids in the top and bottom channels. Hence the gradient is not that steep compared to the other migration devices. But still a stable gradient is observed where the loaded cells to sense the gradient and react accordingly.

Few reasons for collagen layer breakage includes: non uniform collagen dilution, uneven distribution of collagen while loading in the middle channel, Rapid push or pull of fluids while loading, Insufficient curing time. Most of the above limitations can be regulated by gentle loading of the system.

\subsection{Formation of Tubulogenesis inside Microfluidic Device}

HUVEC (2-3 million/ml) cells are loaded on the top chamber and a chemo attractant VEGF (50 $\mathrm{ng} / \mathrm{ml}$ ) is loaded in the bottom chamber. Figure 28 shows loaded top chamber with HUVEC cells and the bottom channel if occupies with VEGF (which is not seen in this picture).

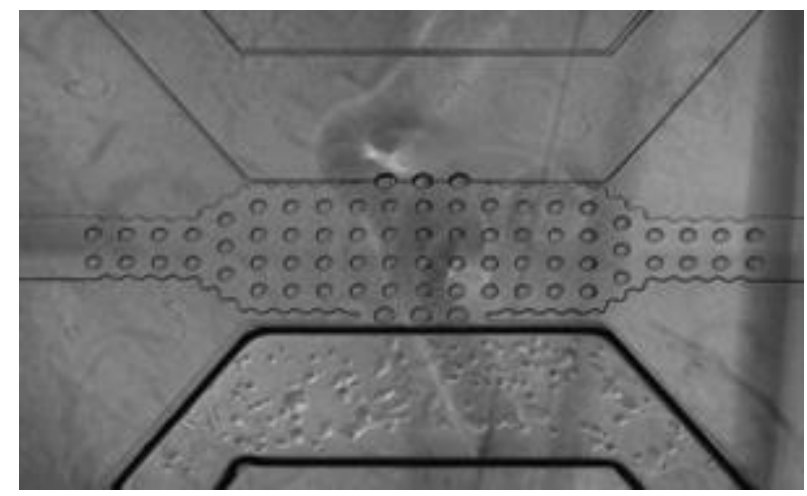

Figure 28 HUVEC cells loaded in the top channel and migration of cells in correspondence to the chemo attractant (VEGF)

The process of cells and chemo attractant loadings are clearly discussed in Chapter-5. Once the device is loaded with cells, it must be placed in an Incubator $\left(37^{\circ} \mathrm{C}\right)$ and the medium is changed 
twice a day. Cells will be completely attached after 3-5 hours of loading and the dead cells need to be washed away, as they may leads to blockage and contamination.

After 4 days from cell loading, tube like structures are observed, from the loading side extended towards the chemo attractant side. This formation of new tubes shows the formation of angiogenesis inside the microfluidic device. Repeated experimental are conducted to analyze the consistent formation of tubing's with and without chemo attractants. Figure 29 shows the control group (without VEGF), where some perfusion of cells can be observed, but there are no signs of tube formation. The total number of cells counted the particular area are 12. When observed from the cell loading side, small change in cell morphology and movement were observed, but there are no traces of tubing formation
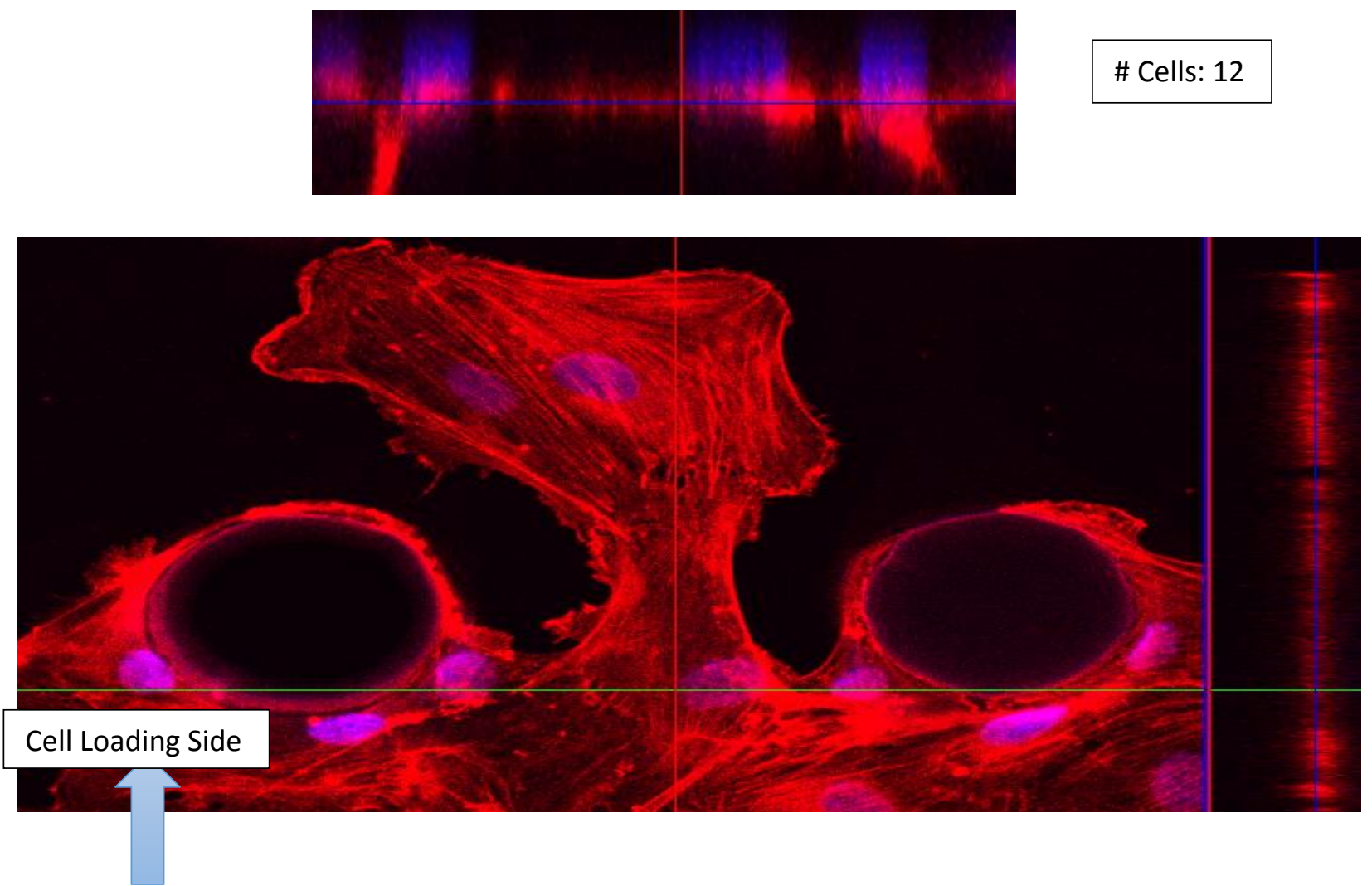

Figure 29 Analysis of control group cells, inside the microfluidic device where there are no traces of tubing formation. 
All the analysis is made using confocal imaging. The 3D imaging is, easy to analyze and quantify the formation of any 3-dimentional structures. The specifications of the confocal microscope and the color indications are discussed below:

The confocal images are at LD Plan-Neofluar 20X magnification : Device of $150 \mu \mathrm{m}$ deep. Color indications are as follows

\section{Red Line : X-axis, Green Line: Y-axis and Blue Line: Z-axis}

The experimental approach group shows that the tube vessel longitudinal and latitudinal diameters, the studied approach for measurement has taken only the focused micro vessels which are quite distinct from the other vessels. It was noted that vessels are most clear and large around the small pillars, and the tubulogenesis along the $\mathrm{Z}$-axis were the result of the gradient profile generated by VEGF.

Immature micro vessels tend to be thin and form slit-like intracellular completely enclosed by a single endothelial cell (seamless capillaries) ${ }^{34}$. Functional micro vessels, however, show larger endothelial tube formations with complete lumens lined by multiple endothelial cells. Confocal images taken at 20x magnification were reconstructed to give a volumetric image in $3 \mathrm{D}$.

The tubogenesis formation is observed in the figures (30) below. Each image is captured at different locations of same device or other devices. The device was subsequently fixed and stained for actin (red) and cell nuclei (blue) to give a composite image that shows the flow occurred through the immune stained endothelial cells. The fraction of patent vessels was calculated using confocal images with 20x magnification. 


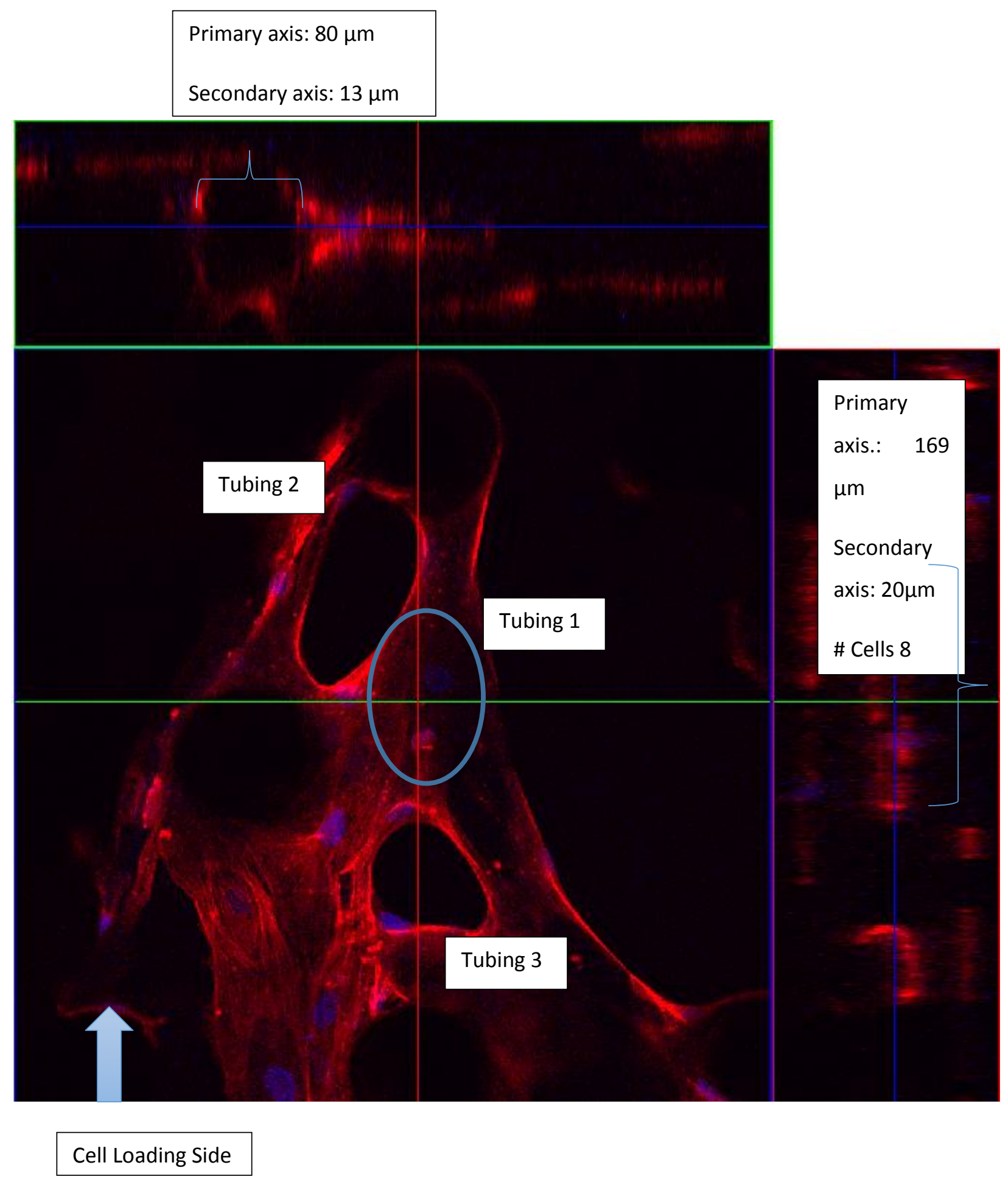

Figure 30 Formation of tubogenesis of HUVEC cells in the experimental group(with VEGF) and having different diameters 
Each location have one or multiple number of tubes and each has its own diameter. The circular tubing formation is shown in the $\mathrm{Z}$ axis, followed by its particular long and short distance diameters.

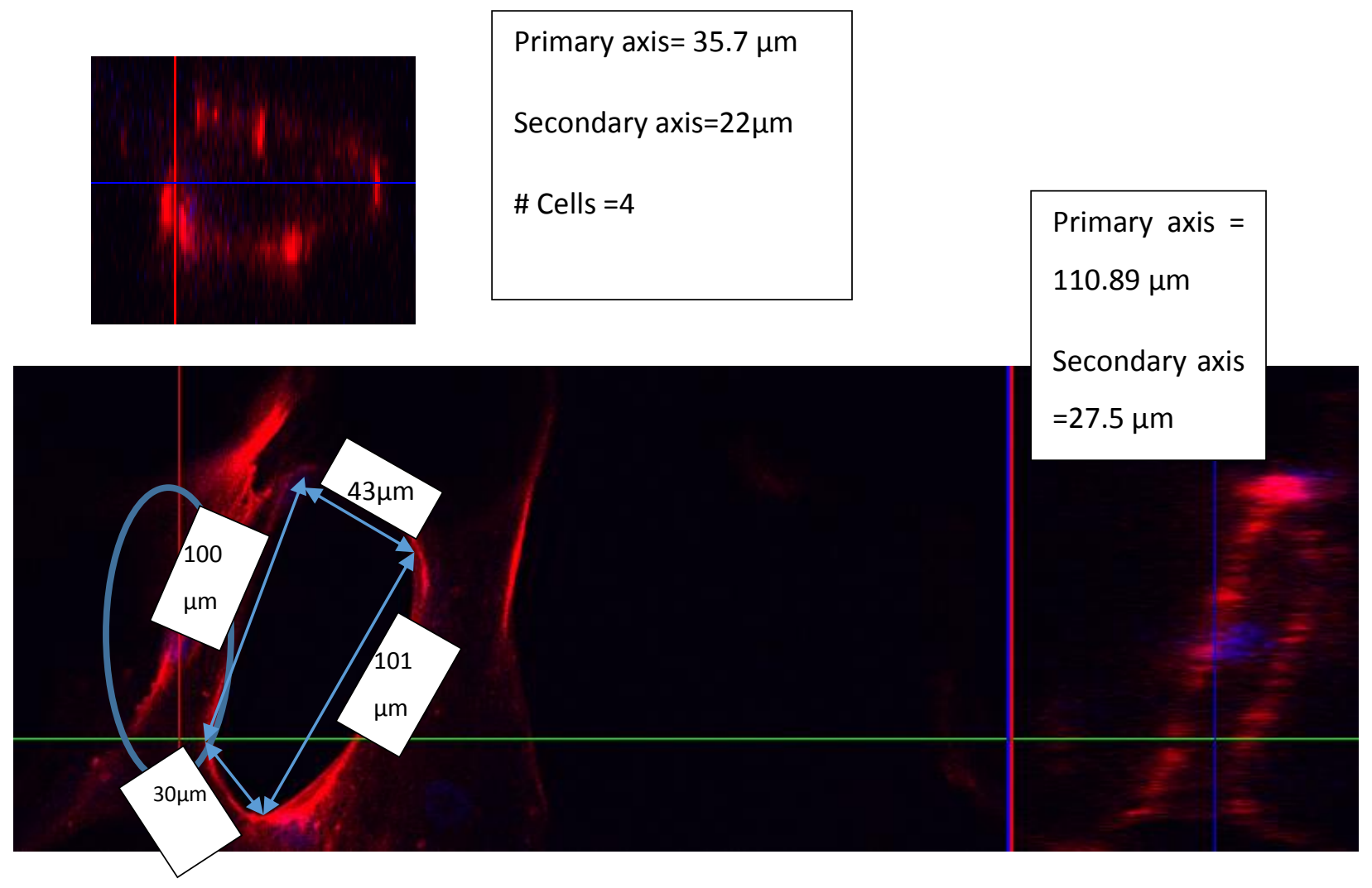

Figure 31 Longitudinal tube formation inside the micro channel, around a micro pillar.

Micro vessels that develop within this microfluidic device recapitulate a number of important angiogenic stages in vivo: cell sprouting, proliferation, tubing formation, branching, and anastomosis ${ }^{35}$. Cellular sprouting processes were dynamic, with the tip cells changing direction and branching from previous sprouts.

When angiogenic factor (VEGF gradient across collagen I) was applied to the ECs cultured in the microfluidic channel, and during several days of culture, the length and projected area of 
sprouting structures into the scaffolds were monitored and quantified. Found that ECs in contact with the VEGF gradient were highly active and rapidly. Sprouted and formed tubes into the scaffold, but cells in contact with the control scaffold were more restrained and demonstrated markedly less sprouting and no tube formation.

Below images shows the formation of tubes into the collagen scaffold at different locations. Many bifurcated cell sprouting were observed which indeed helps for the formation of new tube in other location

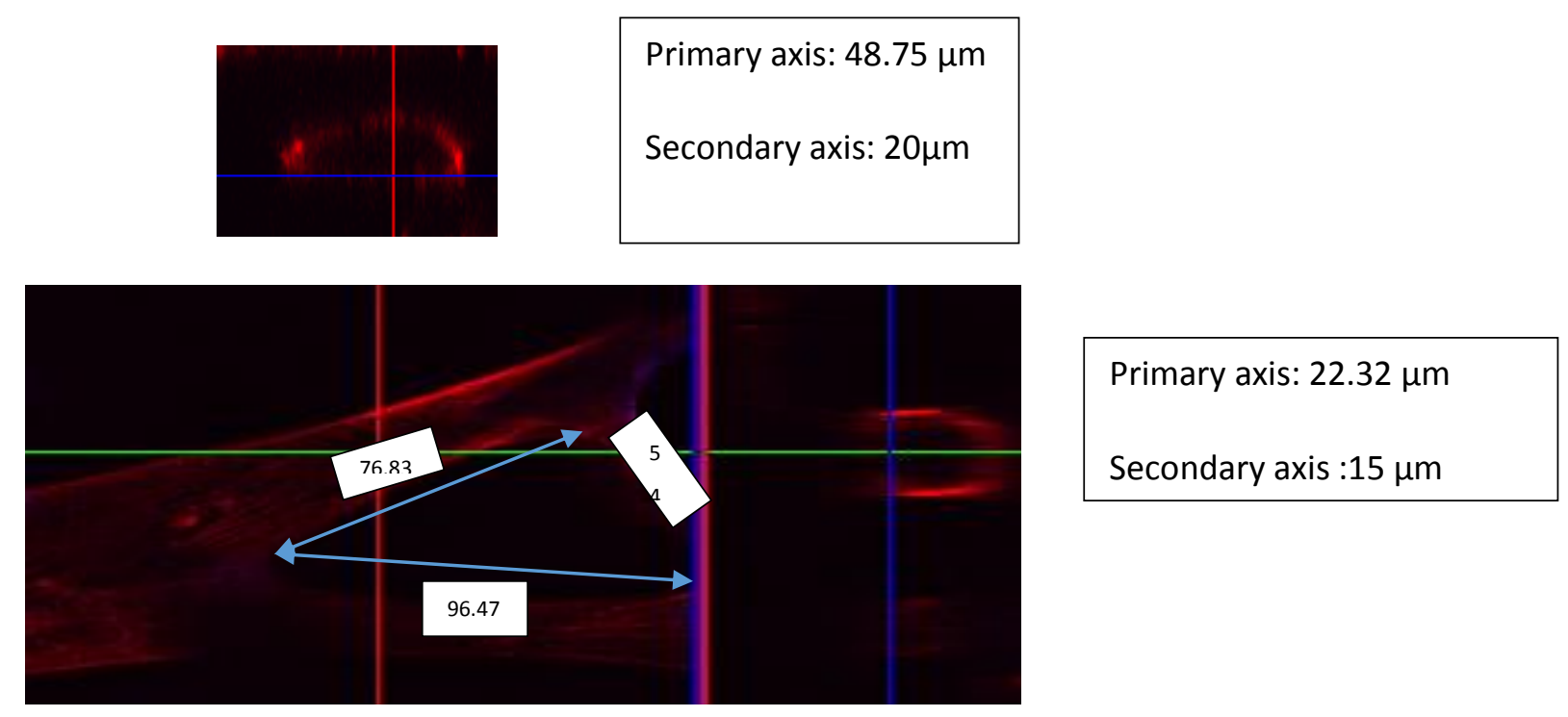

Figure 32 Bifurcation of cell sprouting and formation of tube, around a micro pillar

$3 \mathrm{D}$ culture of cells is essential if we hope to replicate the in vivo situation. Recent advances in microfluidic systems have provided the opportunity to culture HUVEC cells in a precisely controlled microenvironment, including growth factor gradients and flow conditions. Using petri dish approach we can point angiogenesis after couple of days culture, however, it is difficult to 3D image due to their geometry and size. This microfluidic system overcomes some of these limitations in that it enables real-time imaging of cell tubogenesis. 
Few more locational images of the formation of tubogenesis, along with the specifications are shown in the figures below. For better control of angiogenesis, the study of these tubes formation with respect to the generated gradient is mandatory. Although it was difficult to precisely manipulate cells in conventional culture methods, we can try microfluidic culture systems. In particular, more cell types be cultured in a 3D microfluidic system at the same time since multichannel devices have the capacity for seeding different cell types sequentially.

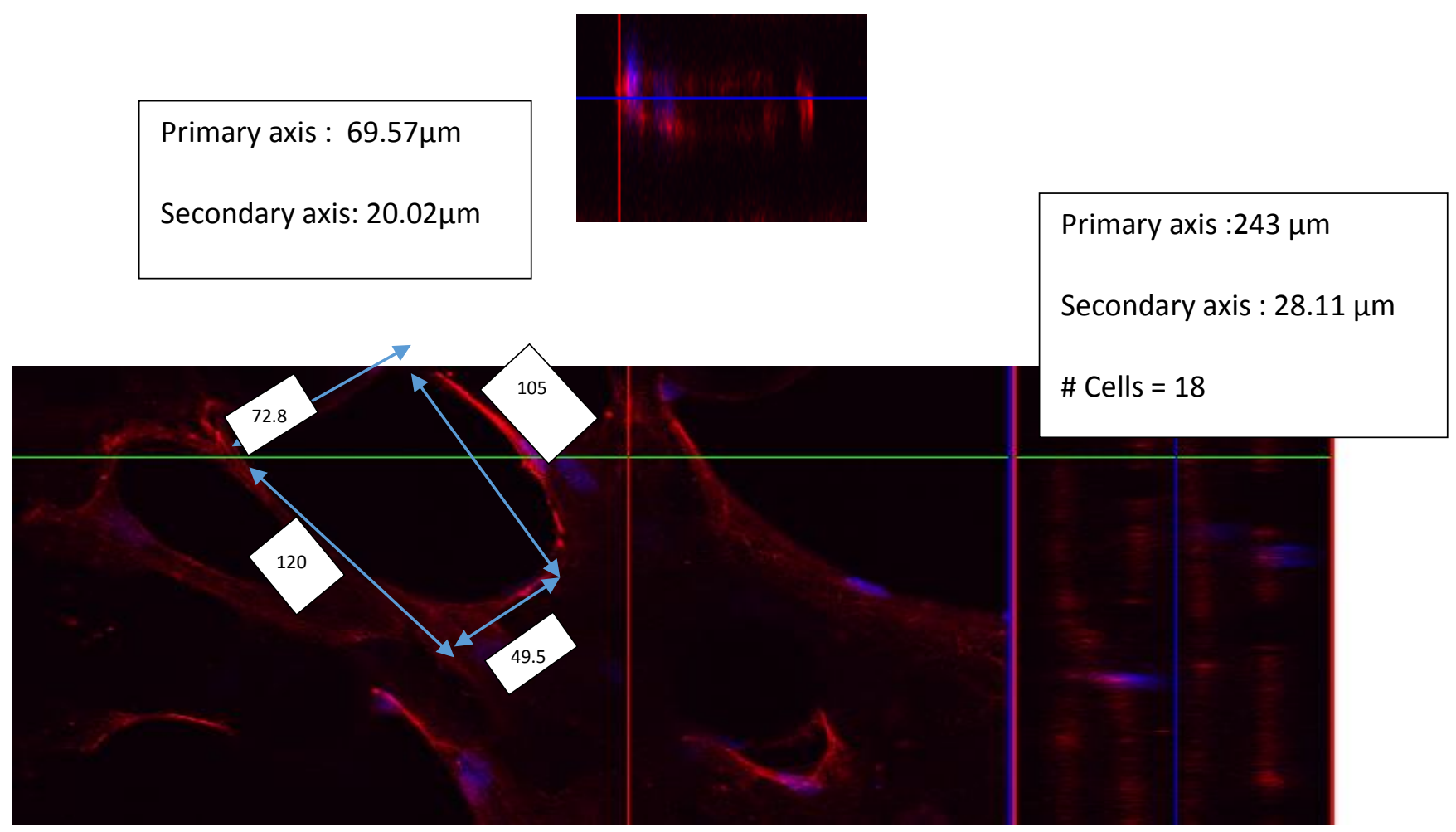

Figure 33 Formation of Lumen, around a micro pillar. 


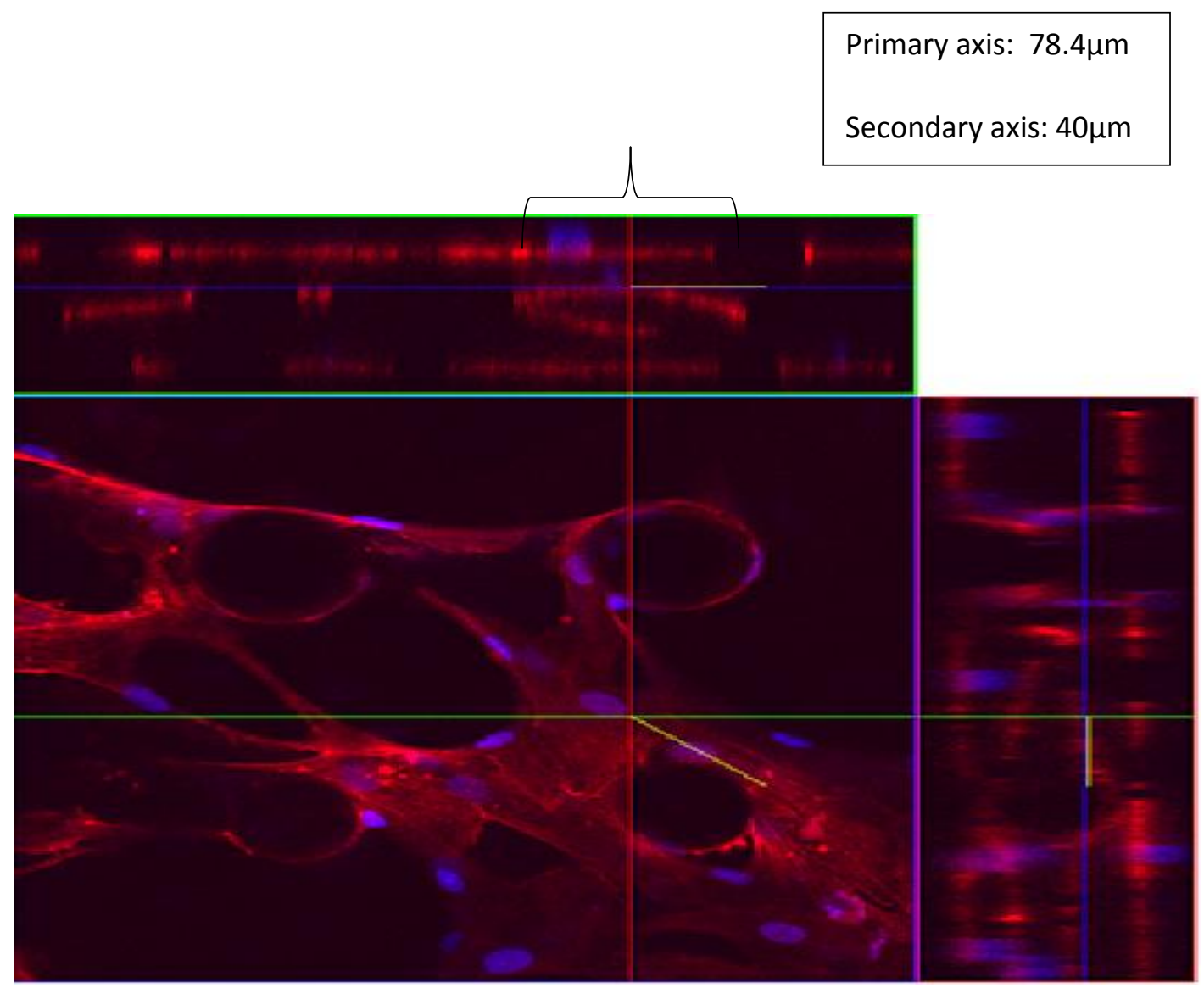

Figure 34 Formation of multiple tubes into the collagen scaffold from the HUVEC monolayer.

In summary, this microfluidic platform provides a versatile platform for designing assays to systematically quantify the effects of the cellular microenvironment on cancer cell motility. Under a chemoattractant, the results of the tube formation inside microfluidic can provide insight into the mechanism of cell sprouting and starting to form new tubings in various locations.Furthermore, the assay enables real-time assessment o capturing 3D cell sprouting and angiogenesis which is very critical step in analysisng thex nature of cancer metastatics. 


\section{CHAPTER 7: CONCLUSION AND FUTURE SCOPE}

A 3D microfluidic based gradient device was designed using AutoCAD and fabricated for real time analysis. In order to mimic the real time micro environment an ECM (Collagen I) was loaded inside the device and tests for stable gradient profile were conducted for longer time. The results showed that microfluidic device is capable enough to generate $3 \mathrm{D}$ gradient, because of the presence of collagen. This device proves as an excellent alternative for cells to grow over longer periods with good viability and staying healthy. The tubing formation with the presence of VEGF was clearly observed using confocal imaging techniques and hence it proves that the microfluidic device is capable of using as an assay for 3D cell migration, tubulogenesis, and angiogenesis.

In future, more experiments are supposed to be conducted on cell angiogenesis and to generate a stable steep gradient without any damage to collagen. Also to present a uniform monolayer of cells to the surface of collagen entrance and to observe proliferation and tube formations of cells from the entrance of collagen to deep into the collagen layer. 


\section{REFERENCES}

${ }^{1}$ Coultas, L.; Chawengsaksophak, K.; Rossant, J. 'Endothelial cells and. VEGF in vascular development', Nature 2005; 438, 937-945

2 Chung, S.; Sudo, R.; Mack, P. J.; Wan, C. R.; Vickerman, V.; Kamm, R. D 'Cell migration into scaffolds under co-culture conditions in a microfluidic platform’,Lab Chip 2009;9, 269-275

${ }^{3}$ Polacheck, W. J.; Charest, J. L.; Kamm, R. D,' Microfluidics for Mammalian Cell Chemotaxis', Proc. Natl. Acad. Sci. 2011; 108, 11115-11120

${ }^{4}$ Lovchik, R. D.; Bianco, F.; Tonna, N.; Ruiz, A.; Matteoli, M.; Delamarche, E.,' Overflow microfluidic networks for open and closed cell cultures on chip', Anal. Chem. 2010; 82, 3936-3942

${ }^{5}$ Park, J. Y.; Takayama, S.; Lee, S. H,' Regulating microenvironmental stimuli for stem cells and cancer cells using microsystems', Integr. Biol. 2010; 2, 229-240

${ }^{6}$ Jeong, G. S.; Kwon, G. H.; Kang, A. R.; Jung, B. Y.; Park, Y.; Chung, S.; Lee, S. H,' Microfluidic assay of endothelial cell migration in 3D interpenetrating polymer semi-network HA-Collagen hydrogel', Biomed. Microdev. 2011; 13, 717-723

7 Yamada, K. M.; Cukierman, E.,' Modeling tissue morphogenesis and cancer in 3D', Cell 2007; 130, $601-610$

${ }^{8}$ Lee, K. H.; Shin, S. J.; Park, Y.; Lee, S. H’ Synthesis of Cell-Laden Alginate Hollow Fibers Using Microfluidic Chips and Microvascularized Tissue-Engineering Applications', Small 2009;5, 1264-1268

9 Auerbach, R.; Auerbach, W.; Polakowski, I. Pharmacol. 'Assays for angiogenesis: a review',Ther. 1991; 51, 1-11

10 Young, Ex. W. K. and Simmons, C. A 'Development of a microfluidic platform for cell cultivation in narrow channels',Lab Chip 2010; 10, 143-160

${ }^{11}$ Van Der Meer, A. D, Poot, A. A., Duits, M. H. G., Feijen, J., and Vermes, I. J'Microfluidic technology in vascularx research', Biomed Biotechnol 200x9; 823148 1-10. 
${ }^{12}$ Sudo, R.; Chung, S.; Zervantonakis, I. K.; Vickerman, V.; Toshimitsu, Y.; Griffith, L. G.; Kamm, R.' Transport-mediated angiogenesis in 3D epithelial coculture',FASEB J 2009; 23, 2155-2164

13 Jain,R.K., Schlenger,K., Hockel,M. and Yuan,F,'Quantitative angiogenesis assays: progress and problems', Nature Med., 3, 1203-1208.

${ }^{14}$ Folman,J .'Tumor angiogenesis, in the Molecular Basis of cancer ',1995; 206-232.

${ }^{15}$ Folkman, J. 'Angiogenesis in cancer,vascular ,rheumatoid and other disease',Nature Med-1995;27-31.

16 S.Patan, L.L.Munn, R.K. Jain, 'Intussusceptive Microvascular growth in a human colon adenocarcinoma xenograp', Microvasc, Res. 1996;260-272.

${ }^{17}$ Rakesh K. Jain, K.Schlender, H.Kichael and Y.Fan,'Quantitative angiogenesis assays:Progress and problems,Nature Medicine,Vol-3,1997;1203.

${ }^{18}$ Gimbrone MAJ, Cotran RS, Leapman SB, Folkman J. 'Tumor growth and neovascularization: an experimental model using the rabbit cornea.',J. Natl. Cancer Inst. 1974;52:413-427.

19 Taylor CM, Weiss JB. 'The chick vitelline membrane as a test system for angiogenesis and antiangiogenesis', Int. J. Microcirc. Clin. Exp. 1984;3:337.

${ }^{20}$ Auerbach R, Akhtar N, Lewis RL, Shinners BL. 'Angiogenesis assays: problems pitfalls', Cancer Metastasis Rev. 2000;19:167-172

${ }^{21}$ Yonekura K, Basaki Y, Chikahisa L, et al. 'UFT and its metabolites inhibit the angiogenesis induced by murine renal cell carcinoma, as determined by a dorsal air sac assay in mice',Clin. Cancer Res.1999;5:2185-2191.

22 Jain RK. Landis award lecture: 'delivery of molecular and cellular medicine to solid tumors',Microcirculation. 1997;4:1-21.

${ }^{23}$ Yuan F, Salehi HA, Boucher Y, Vasthare US, Tuma RF, Jain RK. 'Vascular permeability and microcirculation of gliomas and mammary carcinomas transplanted in rat and mouse cranial windows',Cancer Res. 1994;54:4564-4568.

24 Auerbach R, Kubai L, Sidky YA. 'Angiogenesis induction by tumors, embryonic tissues and lymphocytes',Cancer Res 1976;36:3435-3440

${ }^{25}$ Gimbrone MA, Jr, Leapman SB, Cotran RS, Folkman J. Tumor dormancy in vivo by prevention of neovascularization. J Exp Med 1974;136:261-276 
${ }^{26}$ Passaniti A, Taylor RM, Pili R, Guo Y, Long PV, Haney JA, et al. 'A simple, quantitative method for assessing angiogenesis and antiangiogenic agents using reconstituted basement membrane, heparin and fibroblast growth factor', Lab Invest 1982;67:519-528.

${ }^{27}$ Muthukkaruppan VR, Shinners BL, Lewis R, Park S-J, Baechler BJ, Auerbach R. 'The chick embryo aortic arch assay: a new, rapid, quantifiable in vitro method for testing the efficacy of angiogenic and anti-angiogenic factors in a three-dimensional, serum-free organ culture system', Proc Am Assoc Cancer Res 2000;41:65.

${ }^{28}$ S. J. Lee ,San José State University | Mechanical \& Aerospace Engineering | 2003; Nov 08

${ }^{29}<$ http://www.ee.ucla.edu/ jjudy/classes/ee250a/lectures/EE250A_Lecture_09_ThickFilm_Lithography_ SU-8_files.

${ }^{30}$ MSDS of SU8 from Microchem

${ }^{31}$ Xia, Y.; Whitesides, G. M. "Soft Lithography". Angew. Chem. Int. Ed. Engl. 1998; 37 (5): 551-575.

${ }^{33}$ Ngalim, S. H., Magenau, A., Zhu, Y., Tønnesen, L., Fairjones, Z., Gooding, J. J \& Gaus, K. 'Creating adhesive and soluble gradients for imaging cell migration with fluorescence microscopy', Journal of visualized experiments 2012; JoVE, (74)

${ }^{34}$ Chung S, Sudo R, Maxck PJ, Wan C-R, Vickerman V, et al. 'Cell migration into scaffolds under coculture conditions in a microfluidic platform',Lab on a chip 2009; 269-275.

${ }^{35}$ Folkman J ‘Tumor Angiogenesis’, Advanced Cancer Research 1985; 43: 175-203. 